\title{
Peruvian upwelling plankton respiration: calculations of carbon flux, nutrient retention efficiency, and heterotrophic energy production
}

\author{
T. T. Packard ${ }^{1}$, N. Osma ${ }^{1}$, I. Fernández-Urruzola ${ }^{1}$, L. A. Codispoti ${ }^{2}$, J. P. Christensen ${ }^{3}$, and M. Gómez ${ }^{1}$ \\ ${ }^{1}$ Marine Ecophysiology Group (EOMAR), Universidad de Las Palmas de Gran Canaria, \\ Campus Universitario de Tafira 35017, Las Palmas de Gran Canaria, Spain \\ ${ }^{2}$ Horn Point Laboratory, University of Maryland, 21613-0775 Cambridge, MD, USA \\ ${ }^{3}$ Green Eyes LLC, Easton, MD 21601, USA \\ Correspondence to: T. T. Packard (theodoretrainpackard@gmail.com)
}

Received: 22 October 2014 - Published in Biogeosciences Discuss.: 26 November 2014

Revised: 9 April 2015 - Accepted: 14 April 2015 - Published: 6 May 2015

\begin{abstract}
Oceanic depth profiles of plankton respiration are described by a power function, $R_{\mathrm{CO}_{2}}=\left(R_{\mathrm{CO}_{2}}\right)_{0}\left(z / z_{0}\right)^{b}$, similar to the vertical carbon flux profile. Furthermore, because both ocean processes are closely related, conceptually and mathematically, each can be calculated from the other. The exponent $b$, always negative, defines the maximum curvature of the respiration-depth profile and controls the carbon flux. When $|b|$ is large, the carbon flux $\left(F_{\mathrm{C}}\right)$ from the epipelagic ocean is low and the nutrient retention efficiency (NRE) is high, allowing these waters to maintain high productivity. The opposite occurs when $|b|$ is small. This means that the attenuation of respiration in ocean water columns is critical in understanding and predicting both vertical $F_{\mathrm{C}}$ as well as the capacity of epipelagic ecosystems to retain their nutrients. The ratio of seawater $R_{\mathrm{CO}_{2}}$ to incoming $F_{\mathrm{C}}$ is the NRE, a new metric that represents nutrient regeneration in a seawater layer in reference to the nutrients introduced into that layer via $F_{\mathrm{C}}$. A depth profile of $F_{\mathrm{C}}$ is the integral of water column respiration. This relationship facilitates calculating ocean sections of $F_{C}$ from water column respiration. In an $F_{\mathrm{C}}$ section and in a NRE section across the Peruvian upwelling system we found an $F_{\mathrm{C}}$ maximum and a NRE minimum extending down to $400 \mathrm{~m}, 50 \mathrm{~km}$ off the Peruvian coast over the upper part of the continental slope. Finally, considering the coupling between respiratory electron transport system activity and heterotrophic oxidative phosphorylation promoted the calculation of an ocean section of het-
\end{abstract}

erotrophic energy production (HEP). It ranged from 250 to $500 \mathrm{~J} \mathrm{~d}^{-1} \mathrm{~m}^{-3}$ in the euphotic zone to less than $5 \mathrm{~J} \mathrm{~d}^{-1} \mathrm{~m}^{-3}$ below $200 \mathrm{~m}$ on this ocean section.

\section{Introduction}

Respiration is as ubiquitous in the ocean as the microorganisms that cause it (Seiwell, 1934; Richards, 1957; Lane, 2002). It is controlled by the respiratory electron transport (ETS) activity in eukaryotic mitochondria and prokaryotic cell membranes (Packard, 1969; Packard et al., 1971; Lane, 2005; Nelson and Cox., 2000) and is responsible for the bulk of oceanic $\mathrm{O}_{2}$ consumption (Seiwell, 1937; Redfield et al., 1963; Packard, 1985a). It is driven by the degradation of dissolved and particulate organic carbon, generates $\mathrm{CO}_{2}$ (Redfield et al., 1963), acidifies seawater (Harvey, 1955), and produces energy in the form of ATP (heterotrophic energy production) (Ochoa, 1943; Nelson and Cox., 2000; Madigan et al., 2000). Even in anoxic seawater, respiration degrades organic matter, produces $\mathrm{CO}_{2}$, and generates ATP while reducing nitrogen oxides to $\mathrm{N}_{2}$ or $\mathrm{SO}_{4}^{-}$to $\mathrm{H}_{2} \mathrm{~S}$ (Richards, 1965; Madigan et al., 2000). Plankton community respiration in ocean water columns is a key variable in calculating net community productivity (Ducklow and Doney, 2013) in developing oceanic carbon models, in resolving the autotrophicheterotrophic states of ocean ecosystems (Williams et al., 
2012), and in understanding vertical ocean $F_{\mathrm{C}}$ rates (Giering et al., 2014). The research team led by Sarah Giering (Giering et al., 2014) demonstrated that, contrary to previous efforts (Burd et al., 2010) but in accord with classical oceanographic understanding (Riley, 1951; Richards, 1957; Redfield et al., 1963; Suess, 1980), zooplankton and microplankton (prokaryote and eukaryote) respiration balance vertical carbon flux (Riley, 1951; Eppley and Peterson, 1979; Packard et al., 1988). All these findings support the use of plankton respiration in assessing vertical $F_{\mathrm{C}}$ in the ocean water column. Conceptually, the reciprocal relationship between the water column respiration and the $F_{\mathrm{C}}$, from the ocean's epipelagic zone, is clear (Suess, 1980; Martin et al., 1987). However, describing this reciprocal relationship mathematically, as a function of ocean depth in the forms,

$R=f(z)$ and $F_{\mathrm{C}}=\int_{z_{1}}^{z_{2}} R \mathrm{~d} z$,

was delayed until the helium-tritium studies of Jenkins (Jenkins, 1982, 1984), the sediment trap studies of VERTEX program (Martin et al., 1987), and respiratory electron transport system (ETS) measurements in the Gulf of Maine (Packard and Christensen, 2004). In the latter, microplankton ETS measurements were used to build power function models of respiratory $\mathrm{CO}_{2}$ production $\left(R_{\mathrm{CO}_{2}}\right)$ and $F_{\mathrm{C}}$. Here, we extend this approach to calculate a microplankton respiration section across the Peruvian upwelling system ((Walsh, 1972; Barber et al., 1971) and Fig. 1a) and to model $F_{\mathrm{C}}$ on this transect. We focused our measurements on microplankton because its biomass and metabolism dominate ocean water columns (King et al., 1978; Arístegui et al., 2009; Laufkötter et al., 2013). The section was made at a time of regime change when the Peruvian upwelling system and the El Niño-Southern Oscillation (ENSO) underwent a shift (Santoso et al., 2013). Here we document some of the biological phenomenon that occurred at that time. With the $F_{\mathrm{C}}$ and the $R_{\mathrm{CO}_{2}}$ models we calculate the nutrient retention efficiency (NRE) (Packard and Gómez, 2013; Osma et al., 2014), a new metric that quantifies the ability of an ocean layer to retain its nutrients (Fig. 2c). Conceptually, the NRE is the nutrient remineralization rate within an ocean layer normalized by nutrients entering that layer via carbon flux. Below the euphotic zone, it can be calculated as the inverse of the $F_{\mathrm{C}}$ transfer efficiency (Buesseler et al., 2007; Buesseler and Boyd, 2009), but we show here that it can also be calculated from a profile of plankton respiration. In addition, using different limits to the $F_{\mathrm{C}}$ integration, we calculate the sum of the benthic respiration and carbon burial (Fig. 3a) that occurs on the sea floor. Finally, we use the respiration models and the coupling between ETS activity and oxidative phosphorylation to calculate light-independent heterotrophic energy flow (Karl, 2014). This energy is generated in the form of ATP by ATP synthase, an enzyme motor coupled to a heterotrophic respiratory process such as $\mathrm{O}_{2}$ utiliza- tion or $\mathrm{NO}_{3}^{-}$reduction (Watt et al., 2010; Ferguson, 2010). In all types of respiration, the ATP synthase senses the $\mathrm{pH}$ and electromotive force gradient across the membrane in which the ATP synthase is embedded (Lane et al., 2010), and when the gradient is sufficiently strong $(\sim 225 \mathrm{mV})$, the molecular motor that is the ATP synthase starts its rotary production of ATP (Walker, 1998). Heterotrophic ATP generation in any ecosystem is largely based on exploiting the Gibbs free energy $(\Delta \mathrm{G})$ released during the oxidation of different organic compounds. The biochemistry of ATP and the ETS was unknown in 1925, but even then the idea of capturing biologically useable energy from respiration was appreciated by Lotka (1925). A generation later, Odum built on this concept to describe energy flow in freshwater streams (Odum, 1956). Reviewing this earlier work, Karl recently argued that biological energy production in the ocean should be assessed to provide insight into the variability of ocean productivity (Karl, 2014). Here, we address his concern by calculating Heterotrophic energy production (HEP) in a C-line section (Fig. 2d). This HEP is the energy produced while ATP is generated by respiratory $\mathrm{O}_{2}$ consumption $\left(R_{\mathrm{O}_{2}}\right)$ in the microplankton community composed of phytoplankton, bacteria, archaea, and protozoans in the epipelagic layer and by the $R_{\mathrm{O}_{2}}$ and $\mathrm{NO}_{3}^{-}$reduction in microbial communities of bacteria, archaea, and protozoans in the meso- and bathypelagic waters of the Peruvian current upwelling system.

\section{Methods}

\subsection{Research site}

The site of this Coastal Upwelling Ecosystem Analysis (CUEA) investigation at $15^{\circ} \mathrm{S}$ off Pisco, Peru (Fig. 1) was chosen because the upwelling is strong, persistent, and well known (Wooster, 1961; Fernández et al., 2009). It was the focus of the R/V Anton Bruun cruise 15 (Ryther et al., 1970), the R/V T.G. Thompson Pisco expedition in 1969 (Barber et al., 1978), and others (Wyrtki, 1967; Walsh et al., 1971) before it was the focus of the CUEA-JOINT II program (Brink et al., 1981; Packard, 1981) of which the JASON Expedition was a part (King, 1981; Richards, 1981). However, in spite of the many previous expeditions to this site most of them took place in the austral fall (March-April-May). The JASON76 expedition was unique because it took place in the late winter and austral spring (August, September, October, and November) when the southeast trade winds would be at their strongest (Wooster, 1961). In this way it was thought that the results might be more comparable with results from upwelling studies made in the Northern Hemisphere's springtime upwelling off NW Africa (Codispoti et al., 1982; Minas et al., 1982). The results presented here are from the September 10 to September 24 leg of JASON-76 on board Duke University's Oceanographic ship, R/V Eastward, cruise no. E-5H-67 (Packard and Jones, 1976). 

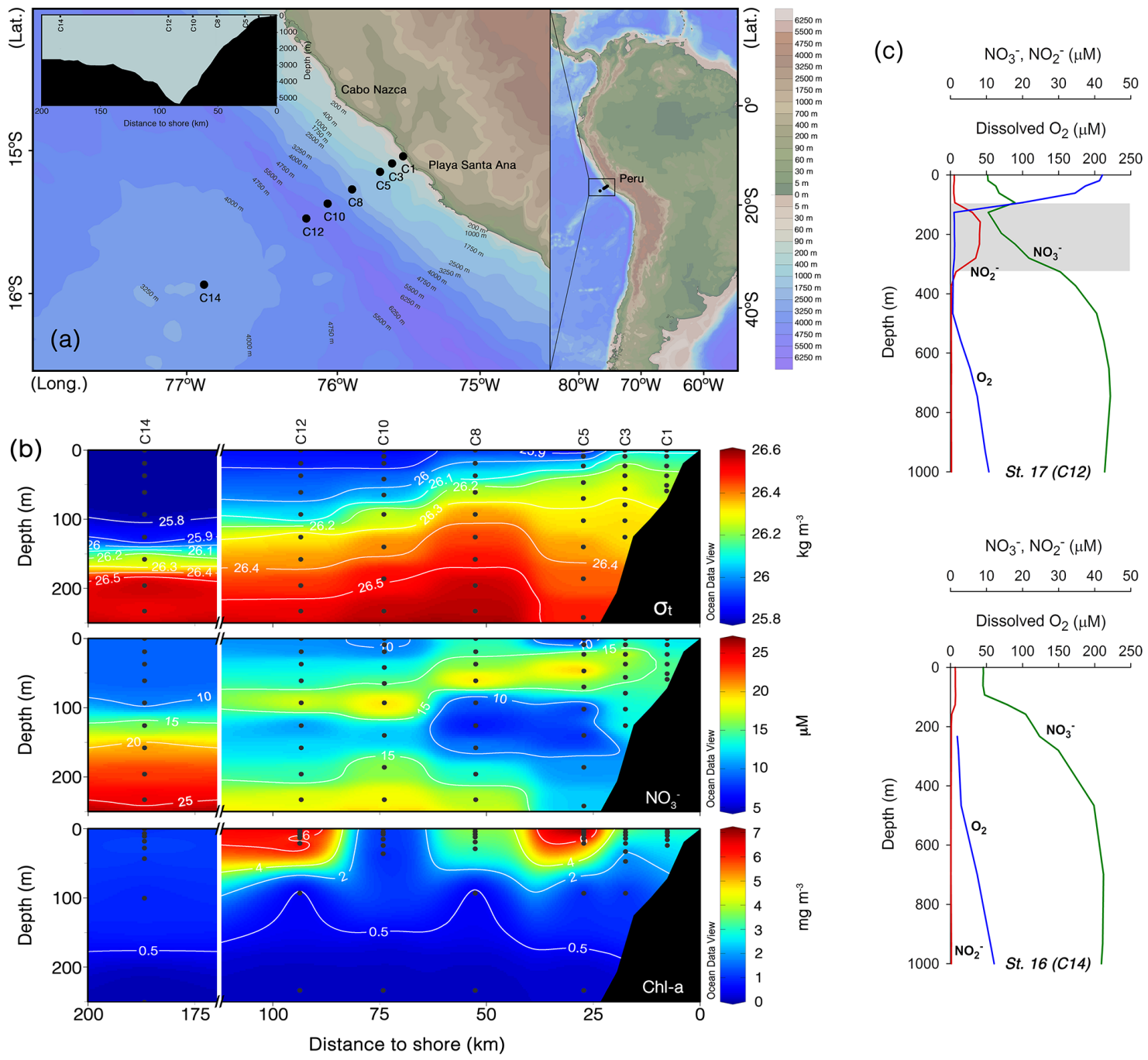

Figure 1. (a) C-line section orthogonal to the Peruvian coast at $15^{\circ} \mathrm{S}$. The innermost $\mathrm{C}$-line position, $\mathrm{C} 1$, was $2.7 \mathrm{~km}$ from the coast between Cabo Nazca and Punta Santa Ana. The outermost position, C14, was located west of the Peru-Chile trench $185.2 \mathrm{~km}$ from the coast. Depth along this transect ranged from $63 \mathrm{~m}$ at $\mathrm{C} 1$ to $4755 \mathrm{~m}$ at $\mathrm{C} 12$. C14 was in $2680 \mathrm{~m}$ of water on the gently rising abyssal plain seaward of the trench (inset upper left). (b) Density $\left(\sigma_{\mathrm{t}}\right), \mathrm{NO}_{3}^{-}(\mu \mathrm{M})$ and phytoplankton chlorophyll $\left(\mathrm{mg} \mathrm{m}^{-3}\right)$ sections along the $\mathrm{C}$-line from $\mathrm{C} 1$ to C14 (top, middle and bottom panels, respectively). All sections represent the upwelling from 13 to 20 September 1976 . Scale brakes avoid interpolation over a $90 \mathrm{~km}$ data gap. The high phytoplankton biomass over the shelf break occurs between $\mathrm{C} 5$ and $\mathrm{C} 8,15$ to $35 \mathrm{~km}$ from the coast. (c) $\mathrm{NO}_{3}^{-}, \mathrm{NO}_{2}^{-}$, and $\mathrm{O}_{2}$ depth profiles through the mesopelagic waters over the trench at $\mathrm{C} 12$ (top) and over the outermost station at $\mathrm{C} 14$ (bottom). The vertical plot at $\mathrm{C} 12$ documents the first step in denitrification (shaded area), $\mathrm{NO}_{3}^{-}$reduction to $\mathrm{NO}_{2}^{-}$, at the foot of the oxycline, in the oxygen minimum zone (OMZ) between 150 and $300 \mathrm{~m}$. In contrast, the vertical profiles at C14, $185 \mathrm{~km}$ off the coast, show the absence of denitrification in mesopelagic waters.

\subsection{Sampling}

All sampling was conducted along the C-line (Fig. 1a) that extended seaward from the coast at position $\mathrm{C} 1$, just south of Cabo Nazca (Pisco), across the deep trench to position C14, $185 \mathrm{~km}$ offshore (Packard, 1981). Hydrographic sections were made at the beginning of the expedition (10-11 September) and again after a lapse of 10 days (20-21 September). The endpoint coordinates were from $15^{\circ} 3.2^{\prime} \mathrm{S}, 75^{\circ} 26.0^{\prime} \mathrm{W}$ to $15^{\circ} 55.8^{\prime} \mathrm{S}, 75^{\circ} 31.4^{\prime} \mathrm{W}$ (Packard and Jones, 1976; Ko- gelshatz et al., 1978). In addition, between 10 and 24 September, productivity stations, that focused on the biological, nutrient chemistry, and biochemical properties at depths where the light was $100,50,30,15,5,1$, and $0.1 \%$ of the surface incident radiation (light depths), were established at C-line positions Packard and Jones (1976). These productivity stations were not made in order along the $\mathrm{C}$-line section, hence the irregularity of the their numerical sequence in Tables 3 7. In addition, some locations along the $\mathrm{C}$-line were occupied 
Table 1. Oceanographic characteristics of Peruvian upwelling C-line stations during Duke University's JASON-76 R/V Eastward cruise no. E-5H-67. Ez depth is the $1 \%$ light level. Original data are in CUEA data reports (Kogelshatz et al., 1978; Packard and Jones, 1976).

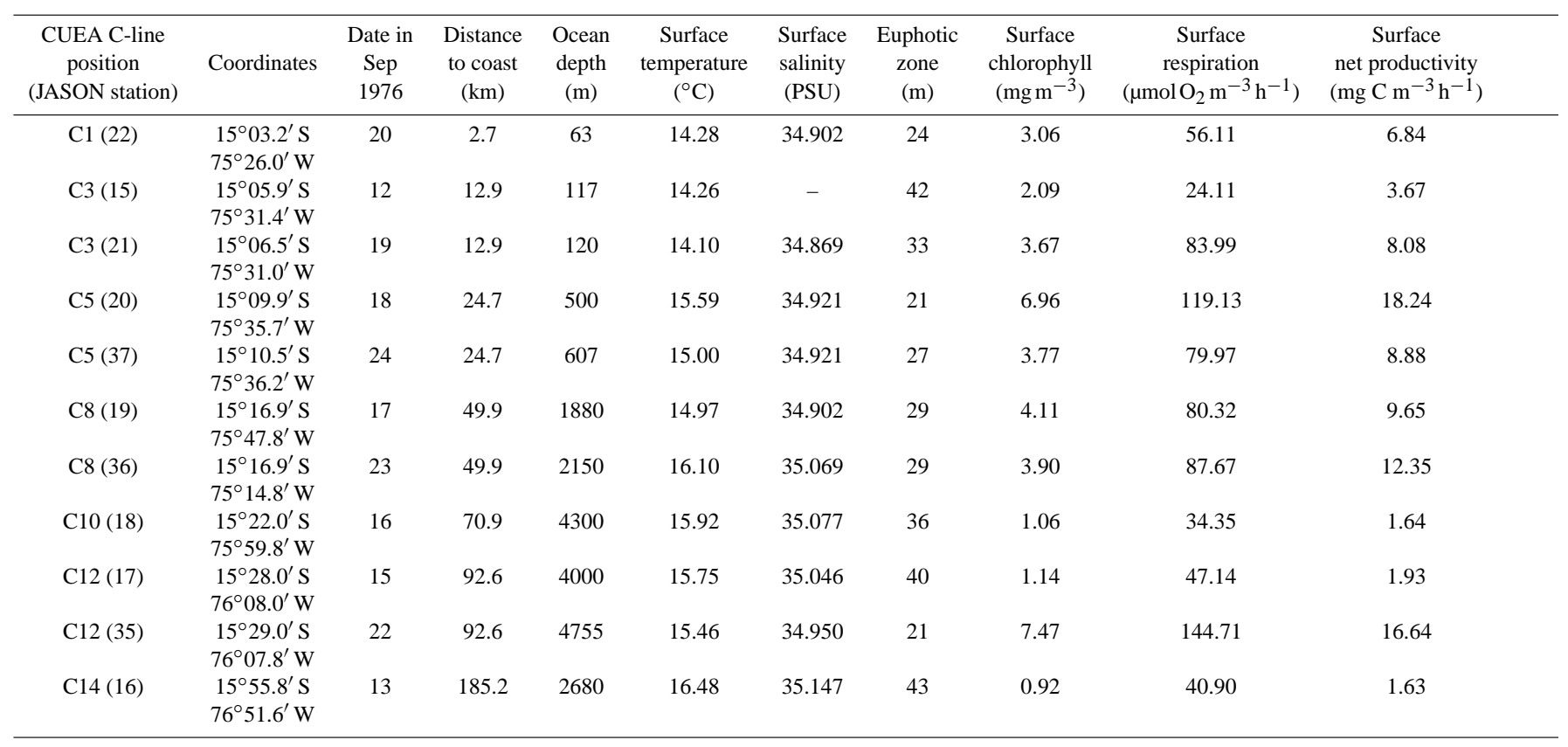

several times. For this reason, as well as to coordinate the results presented here with the results of other CUEA reports (Brink et al., 1981), both the C-line location and the station number are given through the paper. The productivity casts were made each morning before 10:00 LT with $30 \mathrm{~L}$ Niskin PVC bottles to six light depths (1, 5, 15, 30, 50, and $100 \%)$. Each Niskin bottle was flushed at depth in yo-yo fashion both by the action of the ship's roll and by meter oscillations with the winch. On deck it was drained immediately, without prefiltration, into a well-rinsed carboy for subsampling and returned to depth for the next sample. The six samples were taken within $1 \mathrm{~h}$. Subsamples were drawn for phytoplankton productivity, inorganic nutrient salts, (ammonium, reactive phosphorus, $\mathrm{NO}_{3}^{-}, \mathrm{NO}_{2}^{-}$, and silicate), ETS and $\mathrm{NO}_{3}^{-}$reductase activities, and particulate protein (Packard and Jones, 1976). Station coordinates are given in Table 1 . The inorganic nutrient salts, salinity, temperature, and $\mathrm{O}_{2}$ can be found in CUEA data reports 38 and 45 (Hafferty et al., 1978; Kogelshatz et al., 1978). Chlorophyll and phytoplankton productivity $\left({ }^{14} \mathrm{C}\right.$ uptake) are reported in CUEA data report 49 (Barber et al., 1978). The ${ }^{14} \mathrm{C}$ uptake data were calculated on an hourly basis (Table 1 ) from the $24 \mathrm{~h}$ productivity data (Kogelshatz et al., 1978). Light was measured as daily total solar radiation with an Eppley Model 8-48 pyranometer placed above the ship's bridge (Packard and Jones, 1976). Below the mesopelagic zone, the seawater was sampled for ETS activity with $30 \mathrm{~L}$ Niskin PVC bottles down to $2000 \mathrm{~m}$, depending on the depth of the water column (Tables 2 and 3 ).

\subsection{ETS activity, respiratory $\mathrm{O}_{2}$ consumption, $\mathrm{CO}_{2}$ production, and denitrification}

Respiratory ETS activity in the euphotic zone (Ez) was measured according to Kenner and Ahmed (1975) as described in Packard and Williams (1981). In deeper waters it was measured according to (Packard et al., 1971) and multiplied by 3.35 to render the two data sets comparable as explained in Christensen and Packard (1979). Here, ETS activity is used as a direct measure of potential respiration and a proxy for respiration. Both potential respiration and respiration were calculated from the combined ETS data set according to Packard and Christensen (2004) and Packard and Codispoti (2007). Tables 2 and 3 explain the calculations in detail. Table 3 presents the calculations as $R_{\mathrm{O}_{2}}$ in units of $\mu \mathrm{mol} \mathrm{O} \mathrm{O}_{2} \mathrm{~m}^{-3} \mathrm{~h}^{-1}$ for oxic waters. Using ETS activity as a proxy for $R_{\mathrm{O}_{2}}$ requires the selection of a ratio of potential respiration $(\Phi)$ to $R_{\mathrm{O}_{2}}$. Since direct measurements of $R_{\mathrm{O}_{2}}$ can not be made below the euphotic zone, a true calibration can not be made. The $\Phi$ to $R_{\mathrm{O}_{2}}$ ratio should be around 0.5 if $\Phi$ represents $V_{\max }$ of the ETS and standard physiological rates, governed by enzyme activities, operate close to one-half of their potential capacity (Cleland, 1967). With our methodology (Packard and Williams, 1981) and by our analysis (Packard and Christensen, 2004) we calculated a $\Phi$ to $R_{\mathrm{O}_{2}}$ ratio, 0.26 (Table 2 ), that successfully predicted $R_{\mathrm{O}_{2}}$ in the epipelagic and the mesopelagic waters of the Nansen Basin of the Arctic Ocean (Packard and Codispoti, 2007). In that study, $R_{\mathrm{O}_{2}}$ was a long-term average $R_{\mathrm{O}_{2}}$ calculated by the apparent oxygen utilization (AOU)-He-tritium method 
Table 2. Step-by-step calculations of $F_{\mathrm{C}}$ from ETS activity at C-line position C12 (station 35). Potential $R(\Phi), R_{\mathrm{O}_{2}}, \mathrm{~N}_{2}$ production from denitrification $\left(R_{\mathrm{N}_{2}}\right)$ and respiratory $\mathrm{CO}_{2}$ production $\left(R_{\mathrm{CO}_{2}}\right)$ were first determined from temperature-corrected ETS activity values. $\Phi$ is stoichiometrically related to electrons by a factor of $4\left(\mathrm{O}_{2}+4 \mathrm{e}^{-}+4 \mathrm{H}^{+} \rightarrow 2 \mathrm{H}_{2} \mathrm{O}\right) . R_{\mathrm{O}_{2}}$ is 0.26 of $\Phi$ (Packard and Christensen, 2004). $R_{\mathrm{N}_{2}}$ relates to ETS activity according to Codispoti and Packard (1980). Here, denitrifying waters occur between 93 and $233 \mathrm{~m}$ (values in bold). $R_{\mathrm{CO}_{2}}$ was calculated from both $R_{\mathrm{O}_{2}}$ and $R_{\mathrm{N}_{2}}$ (see Sect. 2). Column 7 shows the modeled $R_{\mathrm{CO}_{2}}$ values below the $R$ maximum (13 m), obtained from the depth-normalized power function $\left(R_{\mathrm{CO}_{2}}=R_{\mathrm{m}}\left(z / z_{\mathrm{m}}\right)^{b}\right)$ fitted to the data in Column $6 . F_{\mathrm{C}}$ was determined by integrating either to the bottom $\left(F_{\mathrm{t}-\mathrm{s}}\right.$, Column 8$)$ or to infinity $\left(F_{\infty}\right.$, Column 9$)$. The first represents the $\mathrm{C}$ consumed by $R$ from the Ez $(21 \mathrm{~m})$ to the bottom, while the second includes benthic $R$ and $\mathrm{C}$ burial. The difference between $F_{\infty}$ and $F_{\mathrm{t}-\mathrm{s}}$ equals benthic $R$ and the $\mathrm{C}$ burial rate (Column 10). Column 11 represents the $\mathrm{C}$ flux determined by trapezoidal approximation, which relates to $F_{\mathrm{t}-\mathrm{s}}$ through the regression $F_{\mathrm{t}-\mathrm{s}}=0.85 F_{c_{\text {trap }}}-0.54\left(r^{2}=0.99, p<0.001\right)$.

\begin{tabular}{|c|c|c|c|c|c|c|c|c|c|c|}
\hline $\begin{array}{l}\text { Depth } \\
z \\
(\mathrm{~m})\end{array}$ & $\begin{array}{c}\text { ETS } \\
\text { Activity } \\
\text { (neq } \min ^{-1} \mathrm{~L}^{-1} \text { ) }\end{array}$ & $\begin{array}{c}\Phi \\
\left(\mu \mathrm{molO}{ }_{2}\right. \\
\left.\mathrm{h}^{-1} \mathrm{~m}^{-3}\right)\end{array}$ & $\begin{array}{c}R_{\mathrm{O}_{2}} \\
(\mu \mathrm{mol} \mathrm{O} 2 \\
\left.\mathrm{h}^{-1} \mathrm{~m}^{-3}\right)\end{array}$ & $\begin{array}{c}R_{\mathrm{N}_{2}} \\
\left(\mu \mathrm{molN}_{2}\right. \\
\left.\mathrm{h}^{-1} \mathrm{~m}^{-3}\right)\end{array}$ & $\begin{array}{c}R_{\mathrm{CO}_{2}} \\
(\mu \mathrm{mol} \mathrm{CO} 2 \\
\left.\mathrm{h}^{-1} \mathrm{~m}^{-3}\right)\end{array}$ & $\begin{array}{c}R_{\mathrm{CO}_{2}} \text { modeled } \\
\left(\mu \mathrm{molCO}{ }_{2}\right. \\
\left.\mathrm{d}^{-1} \mathrm{~m}^{-3}\right)\end{array}$ & $\begin{array}{c}F_{\mathrm{C}} \text { to bottom } \\
F_{\mathrm{t}-\mathrm{s}}(\mathrm{mmol} \mathrm{C} \\
\left.\mathrm{d}^{-1} \mathrm{~m}^{-2}\right)\end{array}$ & $\begin{array}{c}F_{\mathrm{C}} \text { to infinity } \\
F_{\infty}(\mathrm{mmol} \mathrm{C} \\
\left.\mathrm{d}^{-1} \mathrm{~m}^{-2}\right)\end{array}$ & $\begin{array}{c}\text { Benthic respiration } \\
\text { and burial } \\
F_{\infty}-F_{\mathrm{t}-\mathrm{s}}\end{array}$ & $\begin{array}{l}\text { C-Flux to bottom } \\
\text { Trap Calc } \\
\left(\mathrm{mmol} \mathrm{Cd}^{-1} \mathrm{~m}^{-2}\right)\end{array}$ \\
\hline 0.5 & 37.10 & 556.56 & 144.71 & - & 102.74 & - & - & - & - & - \\
\hline 3 & 37.81 & 567.14 & 147.46 & - & 104.69 & - & - & - & - & - \\
\hline 5 & 35.69 & 535.39 & 139.20 & - & 98.83 & - & - & - & - & - \\
\hline 9 & 33.65 & 504.68 & 131.22 & - & 93.16 & - & - & - & - & - \\
\hline 13 & 39.19 & 587.87 & 152.85 & - & 108.52 & 1629.67 & - & - & - & - \\
\hline 21 & 15.34 & 230.16 & 59.84 & - & 42.49 & 707.39 & 19.71 & 20.07 & 0.36 & 25.49 \\
\hline 31 & 8.79 & 131.81 & 34.27 & - & 24.33 & 359.18 & 14.68 & 15.04 & 0.36 & 20.16 \\
\hline 93 & 0.44 & - & - & 0.25 & 0.44 & 53.09 & 6.31 & 6.67 & 0.36 & 7.38 \\
\hline 233 & 0.35 & - & - & 0.20 & 0.35 & 10.74 & 3.02 & 3.38 & 0.36 & 3.38 \\
\hline 465 & 0.05 & 0.75 & 0.19 & - & 0.14 & 3.23 & 1.66 & 2.03 & 0.36 & 1.76 \\
\hline 698 & 0.01 & 0.14 & 0.04 & - & 0.03 & 1.59 & 1.14 & 1.50 & 0.36 & 1.20 \\
\hline 930 & 0.02 & 0.23 & 0.06 & - & 0.04 & 0.97 & 0.85 & 1.21 & 0.36 & 0.90 \\
\hline 1395 & 0.01 & 0.21 & 0.05 & - & 0.04 & 0.48 & 0.54 & 0.90 & 0.36 & 0.57 \\
\hline 1860 & 0.01 & 0.13 & 0.04 & - & 0.02 & 0.29 & 0.36 & 0.73 & 0.36 & 0.40 \\
\hline 4755 & - & - & - & - & - & 0.06 & 0 & 0.36 & 0.36 & 0 \\
\hline
\end{tabular}

Table 3. $R_{\mathrm{O}_{2}}$ as $\mu \mathrm{molO}_{2} \mathrm{~h}^{-1} \mathrm{~m}^{-3}$ profiles in the microplankton along the C-line section in September 1976 (roman font values). At the OMZ depths (values in bold), $\mathrm{NO}_{3}^{-}$was the electron donor and $\mathrm{N}_{2}$ was produced during denitrification $\left(R_{\mathrm{N}_{2}}\right.$ as $\left.\mu \mathrm{molN}_{2} \mathrm{~h}^{-1} \mathrm{~m}^{-3}\right)$. C-line position as well as JASON station number (in parentheses) are given. Depth (z) is in meters and $R$ refers to either $R_{\mathrm{O}_{2}}$ or $R_{\mathrm{N}_{2}}$ depending on the font. Calculations are explained in the text.

\begin{tabular}{|c|c|c|c|c|c|c|c|c|c|c|c|c|c|c|c|c|c|c|c|c|c|}
\hline \multirow{2}{*}{\multicolumn{2}{|c|}{$\begin{array}{l}\mathrm{C} 1 \text { (22) } \\
\end{array}$}} & \multicolumn{2}{|c|}{ C3 (15) } & \multicolumn{2}{|c|}{ C3 (21) } & \multicolumn{2}{|c|}{ C5 (20) } & \multicolumn{2}{|c|}{ C5 (37) } & \multicolumn{2}{|c|}{ C8 (19) } & \multicolumn{2}{|c|}{ C8 (36) } & \multicolumn{2}{|c|}{$\mathrm{C} 10$ (18) } & \multicolumn{2}{|c|}{$\mathrm{C} 12$ (17) } & \multicolumn{2}{|c|}{$\mathrm{C} 12(35)$} & \multicolumn{2}{|c|}{ C14 (16) } \\
\hline & & $z(m)$ & $R$ & $z(m)$ & $R$ & $z(m)$ & $R$ & $z(m)$ & $R$ & $z(m)$ & $R$ & $z(m)$ & $R$ & $z(m)$ & $R$ & $z(m)$ & $R$ & $z(m)$ & $R$ & $z(m)$ & $R$ \\
\hline 0.5 & 56.11 & 0.5 & 24.11 & 0.5 & 83.99 & 0.5 & 119.13 & 0.5 & 79.97 & 0.5 & 80.32 & 0.5 & 87.67 & 0.5 & 34.35 & 0.5 & 47.14 & 0.5 & 144.71 & 0.5 & 40.90 \\
\hline 4 & 84.66 & 6 & 34.89 & 5 & 78.92 & 3 & 154.77 & 4 & 86.75 & 4 & 92.40 & 5 & 97.89 & 5 & 35.79 & 6 & 30.65 & 3 & 147.46 & 7 & 34.62 \\
\hline 6 & 44.15 & 9 & 35.86 & 9 & 64.26 & 6 & 130.83 & 7 & 95.81 & 8 & 75.17 & 8 & 88.41 & 8 & 51.17 & 10 & 39.54 & 5 & 139.20 & 11 & 36.09 \\
\hline 10 & 71.05 & 17 & 42.74 & 14 & 43.68 & 9 & 180.26 & 11 & 86.29 & 12 & 54.66 & 12 & 83.74 & 15 & 40.07 & 16 & 29.04 & 9 & 131.22 & 18 & 54.67 \\
\hline 16 & 60.88 & 28 & 30.99 & 21 & 20.57 & 14 & 97.08 & 17 & 77.46 & 19 & 69.74 & 19 & 63.75 & 24 & 87.36 & 26 & 34.38 & 13 & 152.85 & 28 & 44.78 \\
\hline \multirow[t]{9}{*}{24} & 42.41 & 42 & 15.42 & 33 & 12.05 & 21 & 41.27 & 27 & 67.29 & 29 & 45.27 & 29 & 19.16 & 36 & 24.75 & 40 & 29.61 & 21 & 59.84 & 43 & 25.66 \\
\hline & & & & 47 & 3.15 & 93 & 0.48 & 40 & 71.55 & 93 & 1.12 & 44 & 18.08 & 233 & 0.16 & 233 & 0.09 & 31 & 34.27 & 100 & 4.51 \\
\hline & & & & 93 & 1.80 & 233 & 0.67 & & & 233 & 0.48 & 93 & 0.59 & 465 & 0.04 & & & 93 & 0.25 & 250 & 0.73 \\
\hline & & & & & & 465 & 0.09 & & & 465 & 0.62 & 233 & 0.15 & 1860 & 0.02 & & & 233 & 0.20 & 500 & 0.22 \\
\hline & & & & & & & & & & 930 & 0.36 & 465 & 0.04 & & & & & 465 & 0.19 & 750 & 0.11 \\
\hline & & & & & & & & & & 1395 & 0.22 & 698 & 0.03 & & & & & 698 & 0.04 & 1000 & 0.06 \\
\hline & & & & & & & & & & & & 930 & 0.05 & & & & & 930 & 0.06 & 2000 & 0.09 \\
\hline & & & & & & & & & & & & 1395 & 0.06 & & & & & 1395 & 0.05 & & \\
\hline & & & & & & & & & & & & & & & & & & 1860 & 0.04 & & \\
\hline
\end{tabular}

of Jenkins (1982, 1984) as used by W. Roether in Zheng et al. (1997). We have chosen to use the same $\Phi$-to- $R_{\mathrm{O}_{2}}$ ratio of 0.26 here (Tables 2 and 3). $R_{\mathrm{CO}_{2}}$ (Fig. 2a) was then calculated from $R_{\mathrm{O}_{2}}$ using a Redfield ratio $\left(\mathrm{C} / \mathrm{O}_{2}\right)$ of 0.71 from Takahashi et al. (1985). This is the best available way to calculate seawater respiration from our water column ETS measurements.

In waters where respiration is based on using oxides of nitrogen $\left(\mathrm{NO}_{3}^{-}, \mathrm{NO}_{2}^{-}, \mathrm{N}_{2} \mathrm{O}\right.$, or $\left.\mathrm{NO}\right)$ in place of $\mathrm{O}_{2}$, calculations are different. Since microbial respiratory $\mathrm{NO}_{3}^{-}$reduction to nitrogen gas (denitrification) occurs in the water column between 47 and $400 \mathrm{~m}$ between positions C3 to C12 (Garfield et al., 1979; Codispoti and Packard, 1980) (Fig. 2a), Table 3 presents denitrification rates from these depths (shaded numbers) as $R_{\mathrm{N}_{2}}$ in units of $\mu \mathrm{mol} \mathrm{N} \mathrm{m}^{-3} \mathrm{~h}^{-1}$. In these oxygendeficient waters we used a Redfield ratio, $\mathrm{C} / \mathrm{N}_{2}$, from Gruber and Sarmiento (1997). To apply it, one first has to calculate $R_{\mathrm{N}_{2}}$ based on the fact that the ETS for $R_{\mathrm{O}_{2}}$ and $R_{\mathrm{N}_{2}}$ differ only in the terminal electron acceptor (Packard, 1969; Chen and Strous, 2013). This was done in Tables 2 and 3 according to Codispoti and Packard (1980) and Codispoti et al. (2001). The approach has recently been corroborated by Dalsgaard et al. (2012). $R_{\mathrm{N}_{2}}$ in units of $\mu \mathrm{molN} \mathrm{N}_{2} \mathrm{~h}^{-1} 1 \mathrm{~m}^{-3}$ is calculated in Table 2 , column 2 , by multiplying nanoeq $\min ^{-1} \mathrm{~L}^{-1}$ by 60 . The product is equivalent to $\mu \mathrm{mol} \mathrm{e}^{-} \mathrm{h}^{-1} \mathrm{~m}^{-3}$. Then, dividing this by $105 \mathrm{~mol} \mathrm{e}^{-}$per mol $\mathrm{N}_{2}$ yields $R_{\mathrm{N}_{2}}$. The 
Table 4. Power functions for microplankton $R\left(\mathrm{mmolCO}_{2} \mathrm{~d}^{-1} \mathrm{~m}^{-3}\right)$ as functions of normalized depth, $R_{\mathrm{CO}_{2}}=R_{\mathrm{m}}\left(z / z_{\mathrm{m}}\right)^{b}$, where $R_{\mathrm{CO}_{2}}$ is the respiratory $\mathrm{CO}_{2}$ production at any depth $(z), R_{\mathrm{m}}$ is the $R$ maximum $\left(\mathrm{mmolCO}_{2} \mathrm{~d}^{-1} \mathrm{~m}^{-3}\right)$ in the water column, $z / z_{\mathrm{m}}$ is the depth normalized by the depth at $R_{\mathrm{m}}$, and $b$ is the maximum curvature of the power function. Both $z / z_{\mathrm{m}}$ and $b$ are unitless. $\Delta z$ represents the depth range of the $R$ values considered. The table includes the $r^{2}$ from the least-square regression analysis of the $R$ models (SigmaPlot vs. $12.5)$ and the number of data considered $(n)$. The significance level of the regressions is indicated by superscript letters a, b, and c. The last four columns represent the linear regression of the respiration model verification analysis. The slope, the intercept and the $r^{2}$ are given. The $n$ value for each verification analysis is the same as the $n$ used for each $R$ model (column 7). These $R$ models are based on ETS activity data taken during R/V Eastward JASON-76 expedition, along the C-line.

\begin{tabular}{ccccccccccc}
\hline $\begin{array}{c}\text { CUEA C-line } \\
\text { position }\end{array}$ & $\Delta z$ & $z \mathrm{~m}$ & $\begin{array}{c}R_{\mathrm{m}} \\
\left(\mathrm{mmol} \mathrm{CO}_{2}\right.\end{array}$ & $b$ & $r^{2}$ & $n$ & \multicolumn{3}{c}{ Modeled vs. calculated $R_{\mathrm{CO}_{2}}$} \\
(JASON station) & $(\mathrm{m})$ & $(\mathrm{m})$ & $\left.\mathrm{d}^{-1} \mathrm{~m}^{-3}\right)$ & & & & slope & intercept & $r^{2}$ & $n$ \\
\hline C1 (22) & $4-24$ & 4 & 1.538 & -0.355 & 0.864 & 4 & 0.975 & 25.24 & 0.878 & 4 \\
C3 (15) & $17-42$ & 17 & 0.783 & -1.109 & 0.929 & 3 & 1.041 & 20.42 & 0.926 & 3 \\
C3 (21) & $5-93$ & 0.5 & 20.659 & -1.080 & $0.972^{\mathrm{c}}$ & 7 & 1.095 & 45.37 & $0.905^{\mathrm{b}}$ & 7 \\
C5 (20) & $9-465$ & 9 & 2.796 & -1.655 & $0.951^{\mathrm{b}}$ & 6 & 0.890 & 4.25 & $0.996^{\mathrm{b}}$ & 6 \\
C5 (37) & $7-40$ & 7 & 1.596 & -0.192 & $0.873^{\mathrm{a}}$ & 5 & 0.875 & 168.87 & $0.902^{\mathrm{a}}$ & 5 \\
C8 (19) & $4-1395$ & 4 & 3.247 & -1.168 & $0.957^{\mathrm{c}}$ & 10 & 1.348 & 129.78 & $0.686^{\mathrm{a}}$ & 10 \\
C8 (36) & $5-1395$ & 5 & 4.413 & -1.670 & $0.949^{\mathrm{c}}$ & 12 & 0.937 & -44.14 & $0.808^{\mathrm{b}}$ & 12 \\
C10 (18) & $24-1860$ & 24 & 0.946 & -2.051 & $0.962^{\mathrm{a}}$ & 5 & 0.638 & 29.3 & $0.976^{\mathrm{a}}$ & 5 \\
C12 (17) & $0.5-233$ & 0.5 & 3.172 & -0.720 & 0.497 & 7 & 2.596 & -661.42 & 0.339 & 7 \\
C12 (35) & $13-1860$ & 13 & 1.630 & -1.740 & $0.948^{\mathrm{c}}$ & 10 & 0.627 & 10.56 & $0.998^{\mathrm{b}}$ & 10 \\
C14 (16) & $18-2000$ & 18 & 1.183 & -1.624 & $0.968^{\mathrm{c}}$ & 9 & 1.043 & -19.72 & $0.910^{\mathrm{b}}$ & 9 \\
\hline
\end{tabular}

\footnotetext{
a $p<0.05$

b $p<0.001$

c $p<0.0001$
}

Table 5. Microplankton respiration in epipelagic, mesopelagic, and bathypelagic waters along the C-line across the Peruvian current upwelling system at $15^{\circ} \mathrm{S}$. Calculations are based on the $R$ models in Table 4 . Shoreward of $\mathrm{C} 5$, the bottom limits the lower depth boundary. Note the 1000-fold shift in the rates expressed per area (columns 3-7) and per volume (columns 8-10).

\begin{tabular}{|c|c|c|c|c|c|c|c|c|c|}
\hline $\begin{array}{l}\text { CUEA C-line } \\
\text { position } \\
\text { (JASON station) }\end{array}$ & $\begin{array}{c}\text { Ocean } \\
\text { depth } \\
(\mathrm{m})\end{array}$ & $\begin{array}{c}\text { Water column } \\
R \\
\left(\mathrm{mmolC}^{-2} \mathrm{~d}^{-1}\right)\end{array}$ & $\begin{array}{c}\text { Benthic respiration } \\
\text { and } \mathrm{C} \text { burial } \\
(\mathrm{mmol} \mathrm{C} \\
\left.\mathrm{m}^{-2} \mathrm{~d}^{-1}\right)\end{array}$ & $\begin{array}{c}\text { Epipelagic } \\
1-150 \mathrm{~m} \\
(\mathrm{mmolC} \\
\left.\mathrm{m}^{-2} \mathrm{~d}^{-1}\right)\end{array}$ & $\begin{array}{c}\text { Mesopelagic } \\
150-1000 \mathrm{~m} \\
(\mathrm{mmolC} \\
\left.\mathrm{m}^{-2} \mathrm{~d}^{-1}\right)\end{array}$ & $\begin{array}{c}\text { Bathypelagic } \\
1000 \mathrm{~m} \text {-bottom } \\
(\mathrm{mmol} \mathrm{C} \\
\left.\mathrm{m}^{-2} \mathrm{~d}^{-1}\right)\end{array}$ & $\begin{array}{c}\text { Epipelagic } \\
1-150 \mathrm{~m} \\
(\mu \mathrm{mol} \mathrm{C} \\
\left.\mathrm{m}^{-3} \mathrm{~d}^{-1}\right)\end{array}$ & $\begin{array}{c}\text { Mesopelagic } \\
150-1000 \mathrm{~m} \\
(\mu \mathrm{mol} \mathrm{C} \\
\left.\mathrm{m}^{-3} \mathrm{~d}^{-1}\right)\end{array}$ & $\begin{array}{c}\text { Bathypelagic } \\
1000 \mathrm{~m} \text {-bottom } \\
(\mu \mathrm{mol} \mathrm{C} \\
\left.\mathrm{m}^{-3} \mathrm{~d}^{-1}\right)\end{array}$ \\
\hline C1 (22) & 63 & 53.98 & - & 53.98 & - & - & 856.85 & - & - \\
\hline C3 (15) & 117 & 80.32 & 99.23 & 80.32 & - & - & 686.50 & - & - \\
\hline C3 (21) & 120 & 45.81 & 82.99 & 45.81 & - & - & 381.75 & - & - \\
\hline C5 (20) & 550 & 252.48 & 2.60 & 248.99 & 3.49 & - & 1659.96 & 8.72 & - \\
\hline C5 (37) & 607 & 507.91 & - & 162.98 & 344.93 & - & 1086.51 & 754.78 & - \\
\hline C8 (19) & 1880 & 82.10 & 27.37 & 67.58 & 11.46 & 3.07 & 450.51 & 13.48 & 3.49 \\
\hline C8 (36) & 2150 & 153.46 & 0.57 & 150.65 & 2.43 & 0.38 & 1004.36 & 2.85 & 0.33 \\
\hline C10 (18) & 4300 & 1256.23 & 0.09 & 1262.18 & 2.72 & 0.34 & 8414.54 & 3.20 & 0.10 \\
\hline C12 (17) & 4000 & 64.36 & - & 22.28 & 19.57 & 22.51 & 148.55 & 23.02 & 7.50 \\
\hline C12 (35) & 4755 & 318.83 & 0.36 & 314.51 & 3.53 & 0.79 & 2096.75 & 4.16 & 0.21 \\
\hline C14 (16) & 2680 & 318.14 & 1.50 & 310.56 & 6.30 & 1.28 & 2070.43 & 7.41 & 0.76 \\
\hline
\end{tabular}

constant, $105 \mathrm{~mol}^{-}$per mol $\mathrm{N}_{2}$, is the equivalent of the $R_{\mathrm{N}_{2}} /$ ETS ratio in Codispoti and Packard (1980), $2.4 \mu \mathrm{L}$ $\mathrm{O}_{2} \mathrm{~L}^{-1} \mathrm{~h}^{-1} /\left(\mathrm{gN}_{2} \mathrm{~m}^{-3} \mathrm{yr}^{-1}\right)$. The $R_{\mathrm{CO}_{2}}$ calculation is as follows. $R_{\mathrm{CO}_{2}}$ equals $\left[106 / 60 \mathrm{~mol}\right.$ carbon $(\mathrm{mol} \mathrm{N})^{-1} \times$ ETS activity $\left.\left(\mathrm{mol} \mathrm{e}^{-} \mathrm{h}^{-1} \mathrm{~m}^{-3}\right)\right] /\left[105 \mathrm{~mol} \mathrm{e}^{-}(\mathrm{mol} \mathrm{N})^{-1}\right]$. The ratio, 106/60 mol $\mathrm{C}(\mathrm{mol} \mathrm{N})^{-1}$, is the Redfield ratio, mentioned above, for the carbon (as $\mathrm{CO}_{2}$ ) produced during denitrification from $\mathrm{NO}_{3}$ (Gruber and Sarmiento, 1997). Note that both $R_{\mathrm{O}_{2}}$ and $R_{\mathrm{N}_{2}}$, from Table 3 were converted to $R_{\mathrm{CO}_{2}}$ before being used in the Ez part of Fig. 2a.

\subsection{R modeling}

To generate $R$ models as depth functions (Table 4), the ETSbased $R$ was plotted against depths $(z)$ normalized by the depth of the $R$ maximum $\left(z_{\mathrm{m}}\right)$ as we did in Packard and Christensen (2004). From these plots, power functions of the form $R=R_{\mathrm{m}}\left(z / z_{\mathrm{m}}\right)^{b}$ were fitted to the data using SigmaPlot (version 12.5) according to Charland (2002). Note that $R_{\mathrm{m}}$ is the depth of the respiration maximum and $b$, the exponent, is always negative. The exponent $b$ represents the maximum curvature of the respiration-depth profile. Note that $R$ in the 
Table 6. Carbon flux models $F_{\mathrm{C}}$ at C-line positions deeper than $500 \mathrm{~m}$ in the Peruvian upwelling system in Sep 1976. From these models, $F_{\mathrm{C}}$ at four different depths were determined. NRE and $F_{\mathrm{C}}$ transfer efficiency $\left(T_{\text {eff }}\right)$ for the upper mesopelagic waters (150-500 m) are also given. NRE was calculated as $100 \times R_{\mathrm{CO}_{2}} / F_{c_{150}}$, where the $R_{\mathrm{CO}_{2}}$ represents the integrated $R$ between 150 and $500 \mathrm{~m}$; $T_{\text {eff }}$ was calculated as $100 \times F_{\mathrm{C}_{500}} / F_{\mathrm{C}_{150}}$ according to Buesseler et al. (2007).

\begin{tabular}{|c|c|c|c|c|c|c|c|c|c|}
\hline $\begin{array}{l}\text { CUEA C-line } \\
\text { position } \\
\text { (JASON station) }\end{array}$ & $\begin{array}{c}\text { Ocean } \\
\text { depth } \\
(\mathrm{m})\end{array}$ & $\begin{array}{l}\text { Euphotic } \\
\text { zone, } z_{\mathrm{e}} \\
\text { (m) }\end{array}$ & $\begin{array}{c}F_{\mathrm{C}} \\
\text { models }\end{array}$ & $\begin{array}{c}F_{\mathrm{C}} \\
\text { from } z_{\mathrm{e}} \\
(\mathrm{mmol} \mathrm{C} \\
\left.\mathrm{m}^{-2} \mathrm{~d}^{-1}\right)\end{array}$ & $\begin{array}{c}F_{\mathrm{C}} \\
\text { from } 150 \mathrm{~m} \\
(\mathrm{mmol} \mathrm{C} \\
\left.\mathrm{m}^{-2} \mathrm{~d}^{-1}\right)\end{array}$ & $\begin{array}{c}F_{\mathrm{C}} \\
\text { from } 500 \mathrm{~m} \\
(\mathrm{mmol} \mathrm{C} \\
\left.\mathrm{m}^{-2} \mathrm{~d}^{-1}\right)\end{array}$ & $\begin{array}{c}F_{\mathrm{C}} \\
\text { from } 1000 \mathrm{~m} \\
(\mathrm{mmol} \mathrm{C} \\
\left.\mathrm{m}^{-2} \mathrm{~d}^{-1}\right)\end{array}$ & $\begin{array}{c}\text { NRE } \\
150-500 \mathrm{~m} \\
(\%)\end{array}$ & $\begin{array}{c}T_{\text {eff }} \\
150-500 \mathrm{~m} \\
(\%)\end{array}$ \\
\hline C5 (20) & 550 & 21 & $22.07\left(z / z_{\mathrm{e}}\right)^{-0.655}$ & 22.07 & 6.09 & 2.77 & - & 54.6 & 45.4 \\
\hline C8 (19) & 1880 & 29 & $55.25\left(z / z_{\mathrm{e}}\right)^{-0.168}$ & 55.25 & 41.92 & 34.24 & 30.48 & 18.3 & 81.7 \\
\hline C8 (36) & 2150 & 29 & $10.14\left(z / z_{\mathrm{e}}\right)^{-0.670}$ & 10.14 & 3.37 & 1.51 & 0.95 & 55.4 & 44.6 \\
\hline C10 (18) & 4300 & 36 & $14.11\left(z / z_{\mathrm{e}}\right)^{-1.051}$ & 14.11 & 3.15 & 0.89 & 0.43 & 71.8 & 28.2 \\
\hline C12 (35) & 4755 & 21 & $20.07\left(z / z_{\mathrm{e}}\right)^{-0.740}$ & 20.07 & 4.68 & 1.92 & 1.15 & 59.0 & 41.0 \\
\hline C14 (16) & 2680 & 43 & $19.80\left(z / z_{\mathrm{e}}\right)^{-0.624}$ & 19.80 & 5.81 & 2.74 & 1.78 & 52.8 & 47.2 \\
\hline
\end{tabular}

Table 7. HEP as ATP production in epipelagic, mesopelagic, and bathypelagic waters of the C-line section, September 1976. Shoreward of C5 the bottom limits the lower depth boundary.

\begin{tabular}{ccccc}
\hline $\begin{array}{c}\text { CUEA C-line } \\
\text { Location } \\
\text { (JASON station })\end{array}$ & $\begin{array}{c}\text { Ocean } \\
\text { depth } \\
(\mathrm{m})\end{array}$ & $\begin{array}{c}\text { Epipelagic HEP } \\
1-150 \mathrm{~m} \\
\left(\mathrm{~J} \mathrm{~m}^{-3} \mathrm{~d}^{-1}\right)\end{array}$ & $\begin{array}{c}\text { Mesopelagic HEP } \\
150-1000 \mathrm{~m} \\
\left(\mathrm{~J} \mathrm{~m}^{-3} \mathrm{~d}^{-1}\right)\end{array}$ & $\begin{array}{c}\text { Bathypelagic HEP } \\
1000 \mathrm{~m}-\text { bottom } \\
\left(\mathrm{J} \mathrm{m}^{-3} \mathrm{~d}^{-1}\right)\end{array}$ \\
\hline C1 (22) & 63 & 289.63 & - & - \\
C3 (15) & 117 & 232.05 & - & - \\
C3 (21) & 120 & 173.40 & - & - \\
C5 (20) & 550 & 977.93 & 1.19 & - \\
C5 (37) & 607 & 367.24 & 255.11 & - \\
C8 (19) & 1880 & 138.77 & 3.59 & 0.89 \\
C8 (36) & 2150 & 319.41 & 0.80 & 0.03 \\
C10 (18) & 4300 & 2609.16 & 0.89 & 1.23 \\
C12 (17) & 4000 & 43.60 & 4.56 & 0.06 \\
C12 (35) & 4755 & 535.98 & 1.18 & 0.26 \\
C14 (16) & 2680 & 699.88 & 2.51 & - \\
\hline
\end{tabular}

Ez of Fig. 2a is based directly on the ETS measurements (Table 3), while the $R$ in the mesopelagic zone of Fig. 2a is based on the $R$ models in Table 4.

\section{5 $F_{C}$, NRE, and HEP calculations}

The $F_{\mathrm{C}}$ was calculated (Table 2) from depth-normalized water column $R$ (Packard and Christensen, 2004; Packard and Gómez, 2013; Osma et al., 2014). Power functions $\left(R_{\mathrm{CO}_{2}}=\right.$ $\left.R_{\mathrm{m}}\left(z / z_{\mathrm{m}}\right)^{b}\right)$ were selected over logarithmic or exponential functions because they better described the data as previous studies found (Packard and Christensen, 2004; Packard and Codispoti, 2007). Conceptually, planktonic $R_{\mathrm{CO}_{2}}$ in a seawater cube is considered equivalent to the difference between the total $F_{\mathrm{C}_{1}}$ through the top of the cube and total $F_{\mathrm{C}_{2}}$ through the bottom of the cube, where total carbon flux refers to the sum of the dissolved organic carbon (DOC) and the particulate organic carbon (POC) flux. We deduce, on the basis of Craig (1971); Carlson et al. (2010); Hansell et al. (2012), that $R$ based on DOC and lateral POC flux, compared to the $R$ based on the vertical flux of labile POC, is less than $30 \%$ of the total $R$. Note that if organic matter, in any form, is resistant to oxidation (Arrieta et al., 2015), its flux through the water column will not be detected by respiration measurements. The flux will be transparent to our ETS measurements. However, the dissolved organic matter in the ocean, at least, appears to be oxidizable (Arrieta et al., 2015). In all cases, to a first approximation, one can express our conceptual model using the expression, $R_{\mathrm{CO}_{2}}=F_{\mathrm{C}_{1}}-F_{\mathrm{C}_{2}}$. In other words, in the vertical, one-dimensional case, the changes in the $F_{\mathrm{C}}$ between depths in a water column are equal to the $R_{\mathrm{CO}_{2}}$ between those depths. Extrapolating this conceptual model to the deep ocean water column, using continuous mathematics, and assuming seafloor carbon burial to be small, the $F_{\mathrm{C}}$ into the top of a water column $\left(F_{\mathrm{C}_{\mathrm{t}}}\right)$ can be calculated by integrating all the $R$ below the top boundary $\left(z_{\mathrm{t}}\right)$ to the ocean bottom $\left(z_{\mathrm{s}}\right)$.

$$
F_{\mathrm{C}_{\mathrm{t}}}=\int_{z_{\mathrm{t}}}^{z_{\mathrm{s}}} R_{\mathrm{CO}_{2}} \mathrm{~d} z
$$




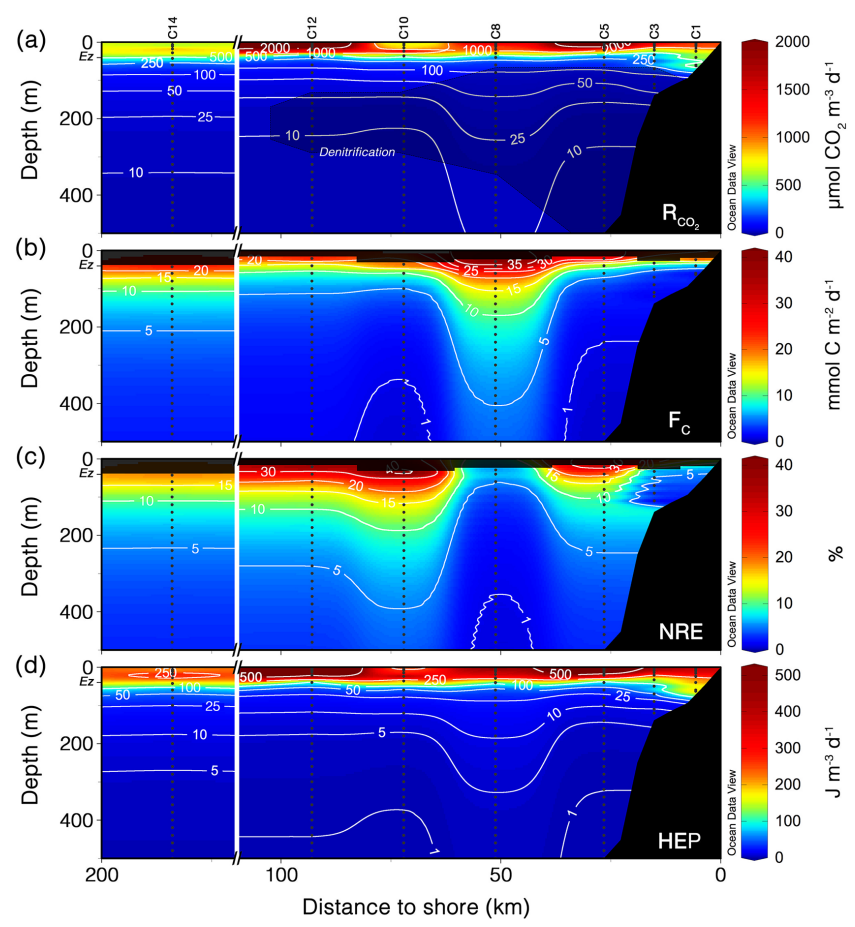

Figure 2. Sections for the upper $500 \mathrm{~m}$ along the C-line. (a) $R_{\mathrm{CO}_{2}}$; the dark shadow delimits denitrification in the OMZ. In the Ez, $R_{\mathrm{CO}_{2}}$ is calculated directly from ETS-based $R_{\mathrm{O}_{2}}$ (Table 3 ). In the mesopelagic waters below, $R_{\mathrm{CO}_{2}}$ is modeled from the respiration equations in Table 4. (b) $F_{\mathrm{C}}$ is calculated by integration of the respiration models (Table 4) to the ocean bottom according to Eqs. (2) and (3). (c) NRE, as a percentage, is determined from models in Tables 4 and 6 as $100 \times\left(R_{\mathrm{CO}_{2}} / F_{\mathrm{C}}\right)$. (d) HEP is either derived directly from ETS activity in the surface waters or from calculated $R_{\mathrm{O}_{2}}$ or $R_{\mathrm{N}_{2}}$ for depths below the Ez (as in Fig. 2a).

All $F_{\mathrm{C}}$ calculations here are based on depth-normalized power functions of $R\left(R_{\mathrm{CO}_{2}}=R_{\mathrm{m}}\left(z / z_{\mathrm{m}}\right)^{b}\right.$, Table 4 ; only if depth is normalized does the equation achieve balance with units of nmol $\left.\mathrm{CO}_{2} \min ^{-1} \mathrm{~L}^{-1}\right)$. For the carbon flux $\left(F_{\mathrm{f}-\mathrm{s}}\right)$ through any depth layer in the water column $\left(z_{\mathrm{f}}\right)$ down to $z_{\mathrm{s}}$, we use Eq. (2) and its integrated version in Eq. (3). Note that these carbon-flux calculations represent the flux at the time the CTD-Niskin cast was made. They are fine-scale calculations of C-Flux.

$$
\begin{gathered}
F_{\mathrm{f}-\mathrm{s}}=\int_{z_{f}}^{z_{s}} R_{\mathrm{CO}_{2}} d z=\int_{z_{f}}^{z_{s}} R_{\mathrm{m}}\left(z / z_{\mathrm{m}}\right)^{b} d z \\
F_{\mathrm{f}-\mathrm{s}}=\left\{R_{\mathrm{m}} /\left[(b+1) z_{\mathrm{m}}^{b}\right]\right\}\left(z_{\mathrm{s}}^{b+1}-z_{\mathrm{f}}^{b+1}\right)
\end{gathered}
$$

Note that $z_{\mathrm{f}}$ is any depth between $z_{\mathrm{t}}$ and $z_{\mathrm{s}}\left(z_{\mathrm{t}} \leqslant z_{\mathrm{f}} \leqslant z_{\mathrm{s}}\right)$ and that $F_{\mathrm{f}-\mathrm{s}}$ is associated with the microplankton respiration, the greater fraction of water column respiration (King et al., 1978).

The NRE is equivalent to $R$ ( $\mathrm{mol} \mathrm{CO} \mathrm{CO}^{-1} \mathrm{~m}^{-3}$ ) within an ocean layer $(\Delta z)$ divided by the $F_{\mathrm{C}}\left(\mathrm{mol} \mathrm{Cd}^{-1} \mathrm{~m}^{-2}\right)$ into the volume of that layer expressed as a percent. Note that the calculation is $(R \times \Delta z) / F_{\mathrm{C}}$. Since the Redfield $\mathrm{N} / \mathrm{C}$ or $\mathrm{P} / \mathrm{C}$ ratio is applied to both parts of the ratio, the $\mathrm{C}, \mathrm{N}$, or $\mathrm{P}$ units cancel out, leaving the ratio unitless. NRE is also related to the carbon flux transfer efficiency (Burd et al., 2010; Buesseler and Boyd, 2009; Buesseler et al., 2007) through the same layer (Packard and Gómez, 2013). For Fig. 2c, it was calculated for $20 \mathrm{~m}$ layers below the Ez to the ocean bottom from the $R$ models in Table 4 and the $F_{\mathrm{C}}$ models in Table 6 .

HEP (Fig. 2d, Table 6) was calculated from $R_{\mathrm{O}_{2}}$ and $R_{\mathrm{N}_{2}}$, derived from the ETS measurements, from the modeled $R_{\mathrm{O}_{2}}$, or from $R_{\mathrm{N}_{2}}$. For oxic seawater HEP $=2 \times 2.5 \times 48 \times R_{\mathrm{O}_{2}}$, where 2 represents the number of electron pairs required to reduce $\mathrm{O}_{2}$ to $2 \mathrm{H}_{2} \mathrm{O}, 2.5$ represents the ATP $/ 2 e^{-}$ratio (Ferguson, 2010), 48 is the $\Delta \mathrm{G}$ in J per mmol of ATP (Alberty and Goldberg, 1992; Moran et al., 2012), and $R_{\mathrm{O}_{2}}$ is the respiratory $\mathrm{O}_{2}$ consumption rate as mmol $\mathrm{O}_{2} \mathrm{~d}^{-1} \mathrm{~m}^{-3}$. For $\mathrm{NO}_{3}^{-}, R$ in anoxic waters, $\mathrm{HEP}=5 \times 1.0 \times 48 \times R_{\mathrm{N}_{2}}$, where 5 is the number of electron pairs required to reduce $\mathrm{NO}_{3}$ to $\mathrm{N}_{2}, 1.0$ is the ATP $/ 2 e^{-}$ratio (van Loosdrecht et al., 1997; Smolders et al., 1994), 48 is the $\Delta \mathrm{G}$ as before, and $R_{\mathrm{N}_{2}}$ is the respiratory $\mathrm{NO}_{3}^{-}$reduction rate as $\mathrm{mmol} \mathrm{N}_{2} \mathrm{~d}^{-1} \mathrm{~m}^{-3}$.

\section{Results}

Oceanographic properties (Table 1) on a C-line transect at $15^{\circ} \mathrm{S}$ across the Peruvian current upwelling system (Fig. 1a) in mid-September of the ENSO transition year, 1976, were measured on the R/V Eastward during the JASON-76 cruise of the CUEA-JOINT II expedition. Classic upwelling (Smith, 1968; Packard et al., 1984; Rykaczewski and Checkley, 2008) was evident during this period. Seawater density $\left(\sigma_{\mathrm{t}}\right)$ and $\mathrm{NO}_{3}^{-}$sloped surface-ward close to the coast (Fig. 1b). From $25 \mathrm{~m}, \sigma_{\mathrm{t}}$ rose from 26.0 to 26.1 , and $\mathrm{NO}_{3}^{-}$ rose from 12 to $16 \mu \mathrm{M}$. As these dense nutrient-rich waters rose, they fertilized the sunlit surface waters at the upwelling center (C3, Brink et al., 1981; MacIsaac et al., 1985), and flowed offshore towards C5 and C8, phytoplankton bloomed to $7 \mathrm{mg} \mathrm{m}^{-3}$ chlorophyll $a$ and $18 \mathrm{mg}$ carbon $\mathrm{h}^{-1} \mathrm{~m}^{-3}$ in productivity (Table 1 and Fig. 1b). The dynamics of this process could be seen in the variability of the Ez depth. It ranged from a low of $21 \mathrm{~m}$ at $\mathrm{C} 5$, the maximum biomass and metabolism position, to twice the depth, $43 \mathrm{~m}$, at the offshore position, C14 (Table 1). Temporal variability was exemplified at the trench position (C12), where over 1 week, the Ez depth decreased from 40 to $21 \mathrm{~m}$. Minimal variability occurred at position C8, where over 6 days, the Ez depth remained at $29 \mathrm{~m}$ (Table 1). In general, a shallow Ez is caused by high plankton biomass with a high potential for metabolism and contrary conditions associated with a deep Ez.

Sea surface $R_{\mathrm{O}_{2}}$ ranged 6-fold from a low of $24.1 \mu \mathrm{mol}$ $\mathrm{O}_{2} \mathrm{~m}^{-3} \mathrm{~h}^{-1}$ at the upwelling center to a high of $144.7 \mu \mathrm{mol}$ $\mathrm{O}_{2} \mathrm{~m}^{-3} \mathrm{~h}^{-1}, 93 \mathrm{~km}$ offshore at the trench position, $\mathrm{C} 12$ (Ta- 

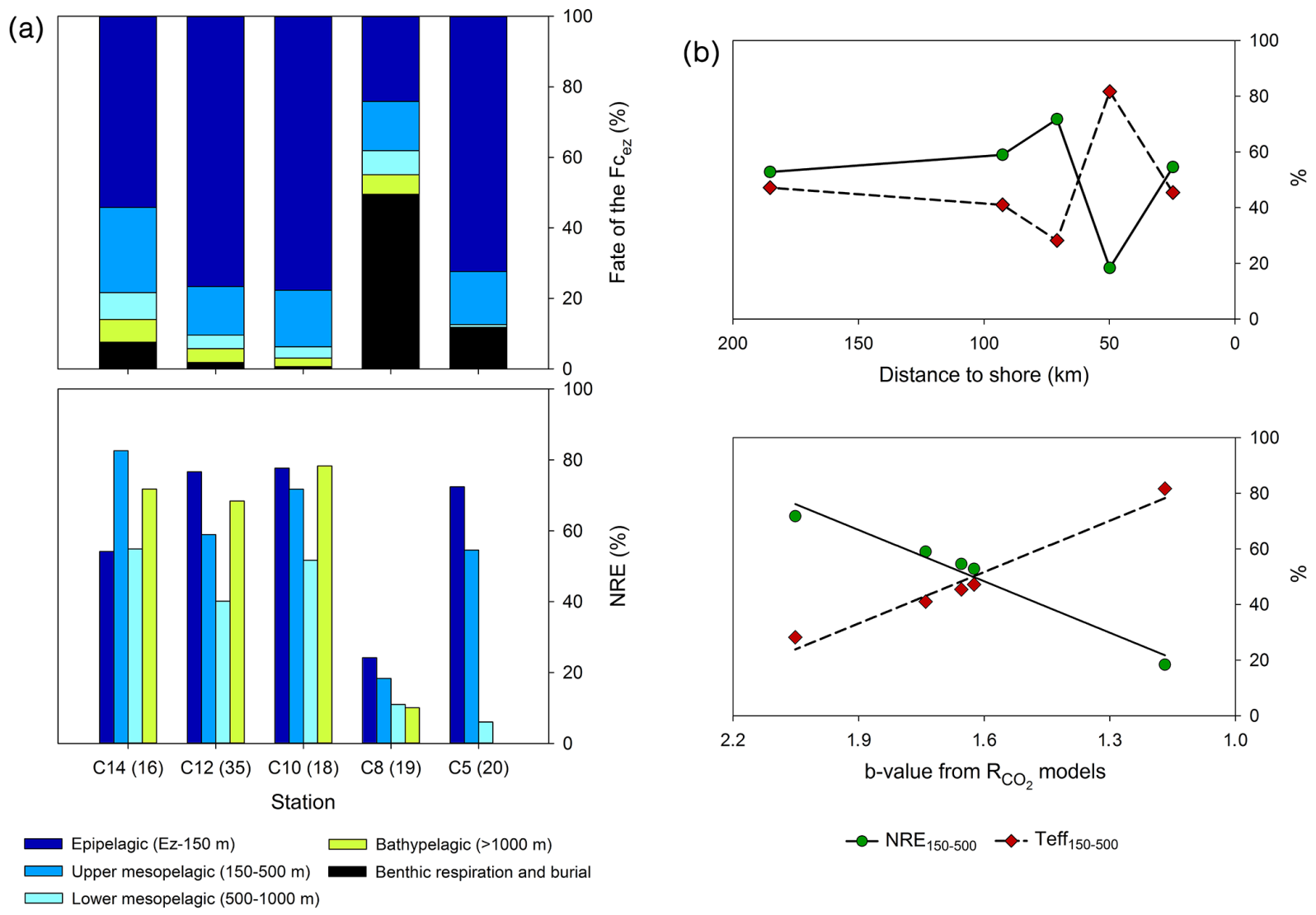

Figure 3. (a) Fate of the carbon fluxing out of the $\mathrm{Ez}\left(F_{\mathrm{C}_{\mathrm{EZ}}}\right)$ into the water column and seafloor below (as a percent of the total flux) along the C-line (top panel). In the water column, the carbon is remineralized through $R$. In the benthos, part of the carbon is remineralized and returned to the water column above and part is buried. The bottom panel shows the different efficiencies with which carbon is remineralized through respiration in four different zones of the water column along the C-line. (b) Top panel: variability of the NRE and the $T_{\text {eff }}$ in the upper mesopelagic waters $(150-500 \mathrm{~m})$ along the C-line. Bottom panel: NRE and $T_{\text {eff }}$ as a function of the maximum curvature $b$ (absolute value) in the $R_{\mathrm{CO}_{2}}$ models from Table 4 .

ble 1). Within days, $R_{\mathrm{O}_{2}}$ could change 3-fold both inshore and offshore (Table 3 ). During the week between $\mathrm{C} 3$ stations 15 and $21, R_{\mathrm{O}_{2}}$ rose from 24.1 to $84.0 \mu \mathrm{mol} \mathrm{O}_{2} \mathrm{~m}^{-3} \mathrm{~h}^{-1}$ and $R_{\mathrm{O}_{2}}$ at $\mathrm{C} 12$ rose from $47.1 \mu \mathrm{mol} \mathrm{O}_{2} \mathrm{~m}^{-3} \mathrm{~h}^{-1}$ (station 17) to $144.7 \mu \mathrm{mol} \mathrm{O} \mathrm{m}^{-3} \mathrm{~h}^{-1}$ (station 35, Table 2). This high respiration $(R)$ at station 35 , occurred in a diatom bloom of Chaetoceros compressus and Ch. lorenzianus. The documentation of such temporal variability in seawater $R_{\mathrm{O}_{2}}$ has only recently begun (Fernández-Urruzola et al., 2014; Osma et al., 2014). Similar increases were seen in the chlorophyll and net productivity at $\mathrm{C} 3$ and $\mathrm{C} 12$ (Table 1 ). The co-occurrence of this rise in $R_{\mathrm{O}_{2}}$, chlorophyll and net productivity suggests seawater $R_{\mathrm{O}_{2}}$ being driven by phytoplankton. Below the immediate sea surface, microplankton $R_{\mathrm{O}_{2}}$ usually increased to a subsurface maximum within the Ez and then decreased dramatically towards the bottom of the $\mathrm{Ez}$ and into the dark ocean below (Tables 2 and 3). $R_{\mathrm{CO}_{2}}$ (Fig. 2a) ranged in the $\mathrm{Ez}$ from $0.4 \mathrm{mmol} \mathrm{CO}_{2} \mathrm{~m}^{-3} \mathrm{~d}^{-1}$ in the upwelling center ( $\mathrm{C} 3$, station 15) to $3 \mathrm{mmol} \mathrm{CO}_{2} \mathrm{~m}^{-3} \mathrm{~d}^{-1}$ at C5, the shelf edge station 20. The lowest epipelagic $R_{\mathrm{CO}_{2}}$
(Table 5) compares with the $R_{\mathrm{CO}_{2}}$ range of $22-27 \mathrm{mmol}$ $\mathrm{CO}_{2} \mathrm{~m}^{-2} \mathrm{~d}^{-1}$ reported recently in eddy-upwelling in the South China Sea (Jiao et al., 2014). In the denitrifying waters, $R_{\mathrm{CO}_{2}}$ was in the $\mu \mathrm{mol}$ range from a low of $4 \mu \mathrm{mol}$ $\mathrm{CO}_{2} \mathrm{~m}^{-3} \mathrm{~d}^{-1}$ at $\mathrm{C} 5$ (station 37) to $133 \mu \mathrm{mol} \mathrm{CO} \mathrm{CO}^{-3} \mathrm{~d}^{-1}$ at $\mathrm{C} 3$ (station 21). In the mesopelagic waters below $500 \mathrm{~m}$ (Table 5), $R_{\mathrm{CO}_{2}}$ ranged from 0.4 to $6.1 \mu \mathrm{mol} \mathrm{CO} \mathrm{CO}_{2} \mathrm{~m}^{-3} \mathrm{~d}^{-1}$ over 1 week at C-line position $\mathrm{C} 8$, at other locations $R_{\mathrm{CO}_{2}}$ fell in between this range. Deeper in the water column, over the trench and beyond, bathypelagic $R_{\mathrm{CO}_{2}}$ ranged from $0.3 \mu \mathrm{mol} \mathrm{CO} \mathrm{CO}^{-3} \mathrm{~d}^{-1}$ at $\mathrm{C} 10$ over the trench to $3.7 \mu \mathrm{mol}$ $\mathrm{CO}_{2} \mathrm{~m}^{-3} \mathrm{~d}^{-1}$ at $\mathrm{C} 8$ over the continental slope (Table 5). Benthic $R_{\mathrm{CO}_{2}}$ and $\mathrm{C}$ burial (Table 5 and Fig. 3a) ranged from a high of $90 \mathrm{mmol} \mathrm{CO}_{2} \mathrm{~m}^{-2} \mathrm{~d}^{-1}$ at $\mathrm{C} 3$, the upwelling center, to a low of $0.09 \mathrm{mmol} \mathrm{CO}_{2} \mathrm{~m}^{-2} \mathrm{~d}^{-1}$ at trench position, C10, with a depth of $4300 \mathrm{~m}$. The $R_{\mathrm{CO}_{2}}$ section in Fig. 2a clearly shows the strength of $R$ and its associated remineralization in the upper $50 \mathrm{~m}$ of the water column and a tongue of high $R$ descending deeper into the water column at position $\mathrm{C} 8,50 \mathrm{~km}$ off the coast. $F_{\mathrm{C}}$ along the C-line tran- 
sect is shown in Fig. 2b. In order to include the inshore stations, $F_{\mathrm{C}}$, in Fig. $2 \mathrm{~b}$, only represents that part of the Cflux that supports the water column respiration. It does not include benthic $R$ and $\mathrm{C}$ burial. To scale our $F_{\mathrm{C}}$ calculations, $F_{\mathrm{C}}$ at $150 \mathrm{~m}$, seaward of $\mathrm{C} 8$, ranging from 3 to $6 \mathrm{mmol}$ $\mathrm{CO}_{2} \mathrm{~m}^{-3} \mathrm{~d}^{-1}$ (Table 6) are comparable to the range of 2.5 to $6.2 \mathrm{mmol} \mathrm{CO}_{2} \mathrm{~m}^{-3} \mathrm{~d}^{-1}$ recently measured at $100 \mathrm{~m}$ by Jiao et al. (2014).

As one would expect with strong $F_{\mathrm{C}}$ at $\mathrm{C} 8$, even at $1000 \mathrm{~m}$, the carbon flux transfer efficiency ( $T_{\text {eff }}$ ) at this station (19) is high and the NRE low (Table 6, Fig. 3b). $T_{\text {eff }}$ between 150 and $500 \mathrm{~m}\left(T_{\text {eff }_{500}}\right)$ is 82 and the NRE is only $18 \%$ (Table 6). Unexpectedly, despite the decrease in $F_{\mathrm{C}}$ throughout the water column at $\mathrm{C} 8$ between 17 and 23 September, $T_{\text {eff }} 500$ only decreased by less than a factor of 2 to $45 \%$ (Table 6 ). The impact on the NRE was greater, increasing 3-fold to $55 \%$ (Table 6). $T_{\text {eff }}$ at other locations ranged from 28 at $\mathrm{C} 10$ to 47 at $\mathrm{C} 14$ (Fig. 3b). In addition to this unique documentation of the temporal variability of $F_{\mathrm{C}}$ from Table 6 , Fig. $2 \mathrm{~b}$ demonstrates its mesoscale spatial variability. That transect shows a maxima occurring throughout the water column, $50 \mathrm{~km}$ from the coast at the upper slope position, C8. As Table 5 and Fig. 3a show, the benthic $R$ and burial are also high at this location. Figure $3 \mathrm{~b}$ highlights the importance of the maximum curvature of the respiration-depth profile. As $|b|$ increases towards 2 the NRE increases towards $70 \%$ and the $T_{\text {eff }}{ }_{50-500}$ decreases towards $30 \%$.

HEP in the Ez (Fig. 2d and Table 7) ranges from a high of $555 \mathrm{~J} \mathrm{~d}^{-1} \mathrm{~m}^{-3}$ at the $R$ maximum at $\mathrm{C} 5$ (station 20) to a low of $69 \mathrm{~J} \mathrm{~d}^{-1} \mathrm{~m}^{-3}$ at the bottom of the $\mathrm{Ez}$ at $\mathrm{C} 3$ (station 21). It drops slightly over the continental slope, but further offshore over the trench (C12) high values of $880 \mathrm{~J} \mathrm{~d}^{-1} \mathrm{~m}^{-3}$ can be found (Fig. 2d). In the far offshore, the Ez HEP only reaches values of $315 \mathrm{~J} \mathrm{~d}^{-1} \mathrm{~m}^{-3}$. As an example of low HEP values, at $4755 \mathrm{~m}$ in the trench it decreases to $0.02 \mathrm{~J} \mathrm{~d}^{-1} \mathrm{~m}^{-3}$. Thus the range of HEP by all the respiratory ETS and oxidative phosphorylation coupling in the microplankton of this part of the Peruvian current upwelling system spans 4 orders of magnitude from $0.02 \mathrm{~J} \mathrm{~d}^{-1} \mathrm{~m}^{-3}$ in the abyssopelagic waters of the trench to $880 \mathrm{~J} \mathrm{~d}^{-1} \mathrm{~m}^{-3}$ in the Ez above. This is the first time such calculations have been made. Integrating the epipelagic HEP (Table 7) over the upper $150 \mathrm{~m}$ yields a range from a low of $6.6 \times 10^{-3} \mathrm{MJ} \mathrm{d}^{-1} \mathrm{~m}^{-2}$ to a high of $0.39 \mathrm{MJ} \mathrm{d}^{-1} \mathrm{~m}^{-2}$, averaging $0.09 \mathrm{MJ} \mathrm{d}^{-1} \mathrm{~m}^{-2}$. This average HEP is only $0.7 \%$ of the average solar radiation $\left(13.5 \pm 4.0 \mathrm{MJ} \mathrm{d}^{-1} \mathrm{~m}^{-2}\right)$ at the C-line sea surface between 12 and 24 September during the JASON-76 cruise (Packard and Jones, 1976).

\section{Discussion}

Here we have demonstrated the calculation of $R_{\mathrm{CO}_{2}}, F_{\mathrm{C}}$, NRE, and HEP in an ocean section from microplankton ETS activity measurements. We have previously explained how ocean water column $R_{\mathrm{CO}_{2}}$ determines $F_{\mathrm{C}}$ of labile organic matter by oxidizing sinking POC and mineralizing phosphate and nitrate (Osma et al., 2014; Packard and Codispoti, 2007). Fig. $3 \mathrm{~b}$ shows that the maximum curvature of the respirationdepth profile determines NRE as well as $F_{\mathrm{C}}$ transfer efficiency. The offshore $R_{\mathrm{CO}_{2}}$ section (Fig. 2a) shows the variability of $R$ with depth and location in the upwelling area. Fig. 2a also shows how seawater respiration is displaced seaward to $\mathrm{C} 8$ from the chlorophyll maximum at C5 (Fig. 1b). The $F_{\mathrm{C}}$ section (Fig. $2 \mathrm{~b}$ ) demonstrates the power of using $R$ to calculate spatial variability of $F_{\mathrm{C}}$ by revealing an $F_{\mathrm{C}}$ maximum over the upper part of the continental slope. The NRE section (Fig. 2c) reveals its inverse relationship to $F_{\mathrm{C}}$ as well as its variability in the water column. This ability of the water column to retain nutrients would not have been detected without the original ETS activity profiles. The HEP section (Fig. 2d), showing the energy production by the ATPases in microbial mitochondrion and plasmalemma membranes of bacteria and archaea in the water column, is a new representation of ATP production in oceanographic analysis. Because a major purpose of all forms of respiration is to make ATP, HEP should reflect $R_{\mathrm{CO}_{2}}$ in any given section or profile. The similarity of the $R_{\mathrm{CO}_{2}}$ pattern in Fig. 2a and the HEP pattern in Fig. 2d confirms this.

Ocean $R_{\mathrm{CO}_{2}}$ filters sinking labile POC and should vary inversely with benthic $R$ and carbon burial. However, the relationship between the two variables is more complicated (Figs. 2a and 3a). We can see this in the $R$ maximum $50 \mathrm{~km}$ off the Peruvian coast at $\mathrm{C}$-line position $\mathrm{C} 8$. One might expect low benthic $R$ and carbon burial here (Table 5), but that is not the case (Fig. 3a). From the difference between integrating the $R$ function (Eq. 2) to infinity and integrating it to the ocean bottom $(z=s)$, we calculate a high level of benthic $R$ and carbon burial (Fig. 3a). The minimum NRE at $\mathrm{C}$-line position, $\mathrm{C} 8$, in Fig. 2c explains this discrepancy. The delivery of labile POC to the bottom depends not directly on $F_{\mathrm{C}}$, but on the ratio of the water column $R$ (Fig. 2a) to $F_{\mathrm{C}}$ (Fig. 2b). Recent studies of the organic carbon preservation on the upper parts of the Peruvian continental slope (Dale et al., 2015) support these calculations of high carbon burial (Fig. 3a.) They find high burial rates at depths between 200 and $400 \mathrm{~m}$ (the upper part of the Peruvian continental slope) and attribute it to the anoxia overlying these sediments. A Cline section of the $T_{\text {eff }}$, the difference between NRE and 1, would have revealed a $T_{\text {eff }}$ maximum at $\mathrm{C} 8$. One can deduce this from Fig. 3b (lower panel).

ETS measurements can be used not only to calculate $F_{\mathrm{C}}$, NRE, and HEP, but also to calculate biological heat production (Pamatmat et al., 1981), age, and flow rates of deep and bottom waters (Packard, 1985a). In anoxic waters, if the background chemistry (Richards, 1965) is known, ETS measurements provide proxy rate measurements for denitrification (Packard, 1969; Codispoti and Packard, 1980; Dalsgaard et al., 2012), $\mathrm{NO}_{2}^{-}$production, nitrous oxide production, and sulfide production (Packard et al., 1983), and even for iron 
and magnesium reduction rates (Lane et al., 2010). All of these types of microbial metabolism are different forms of $R$, but they are controlled by the same basic respiratory ETS with NADH dehydrogenase (Complex 1) as the common gatekeeper. Furthermore, because the energy generation of nitrification is based on a variation of this ETS, an ETS measurement is also likely a proxy for nitrification.

HEP, as ATP generation in the ocean water column, could have been calculated from $R_{\mathrm{O}_{2}}$ since 1943, the time the Nobelist Severo Ochoa first established the connection between ATP production and $R$ (Ochoa, 1943). However, until now, calculations of biological energy production (shown in Fig. 2d), including HEP, in the ocean had not been made (Karl, 2014). Now the time is appropriate to make such calculations with recent research (Lane, 2002, 2005, 2009; Wilson et al., 2012; Chen and Strous, 2013), documenting the ubiquity of respiratory ETS in the biosphere, how it relates to $R_{\mathrm{O}_{2}}$, to all other ocean respiratory processes, and to HEP as ATP production. As we have seen above, HEP and $R_{\mathrm{CO}_{2}}$ in the Peruvian upwelling system have similar time and space distributions (Fig. $2 \mathrm{a}$ and d). The small difference in the ATP $/ 2 e^{-}$relationships between oxidative phosphorylation and the rate of electron transfer in aerobic metabolism and denitrification has minimal impact. In aerobic metabolism, the ATP $/ 2 e^{-}$ratio is 2.5 and in denitrifying microbes ATP $/ 2 e^{-}$is 1.0 (van Loosdrecht et al., 1997; Smolders et al., 1994). At the rate anammox research is progressing (Dalsgaard et al., 2012), its relative contribution will soon be known, too. In any case, less ATP should be produced in anoxic waters resulting in a lower HEP. It will be interesting in the future to look for this difference by comparing HEP offshore sections made through oxic and anoxic sectors of upwelling systems.

\section{Conclusions}

Organic carbon fluxes are critical components of reliable carbon budgets, but they are so difficult to measure that rarely can enough measurements be amassed to construct a synoptic section of $F_{\mathrm{C}}$. Here, from plankton respiration models, we present an original mode of calculating $F_{\mathrm{C}}$ sections as well as benthic respiration and carbon burial. We reveal the importance of plankton respiration in determining the capacity of a plankton community in retaining water column nutrients, develop the concept of NRE, and demonstrate NRE variability in an ocean section. In addition, we show that the curvature of the respiration profile (the exponent $b$ of the power function) controls both the NRE and $F_{\mathrm{C}}$. Finally, we use respiration to calculate the heterotrophic energy production (HEP) and the rate of ATP generated by plankton metabolism, and find an HEP maximum over the shelf break on the upper part of the Peruvian continental slope.
Acknowledgements. We thank J. Ammerman, R. T. Barber, D. Blasco, R. C. Dugdale, N. Garfield, and J. Kogelschatz for their collaboration. D. Bourgault uncovered the role of depth normalization. The suggestions from the three reviewers led to many improvements in this paper and for their diligence we are thankful. NSF (USA) grant OCE 75-23718A01 (TTP) funded JASON-76. The Basque Government (NO and I F-U), MEC (Spain) project BIOMBA, CTM2012-32729/MAR (MG), and CIE (Canary Islands): Tricontinental Atlantic Campus (TTP) funded the analysis.

Edited by: G. Herndl

\section{References}

Alberty, R. and Goldberg, R.: Standard thermodynamic formation properties of adenosie $5^{\prime}$-triphosphate series, Biochemistry, 31, 10610-10615, 1992.

Arístegui, J., Gasol, J., Duarte, C., and Herndl, G.: Microbial oceanography of the dark ocean's pelagic realm, Limnol. Oceanogr., 54, 1501-1529, 2009.

Arrieta, J., Mayol, E., Hansman, L., Herndl, G., Dittmar, T., and Duarte, C.: Dilution limits dissolved organic carbon utilization in the deep ocean, Science, 1258955, doi:10.1126/science.1258955, 2015.

Barber, R., Dugdale, R., MacIsaac, J., and Smith, R.: Variations in phytoplankton growth associated with the source and conditioning of upwelling water, Investig. Pesq., 35, 171-193, 1971.

Barber, R., Huntsman, S., Kogelschatz, J., Smith, W., and Jones, B.: Coastal Upwelling Ecosystems Analysis. Data Report 49. Carbon, Chlorophyll and Light Extinction from JOINT II, vol. 49, CUEA Data Rep., 1978.

Brink, K., Jones, B., Vanleer, C., Mooers, C.,Stuart, D., Stevenson, M., Dugdale, R., and Heburn, G.: Physical and biological structure and variability of the upwelling center off Peru at $15^{\circ} \mathrm{S}$, during March 1977: Coastal Upwelling. Coastal and estuarine sciences 1, vol. xi-xiii, ed Richards FA (American Geophysiscal Union, Wasington DC), 473-495, 1981.

Buesseler, K. and Boyd, P.: Shedding light on precesses that control particle export and flux attenuation in the twilight zone of the open ocean, Limnol. Oceanogr., 54, 1210-1232, 2009.

Buesseler, K., Lamborg, C., Boyd, P., Lam, P., Trull, T., Bidigare, R., Bishop, J., Casciotti, K., Dehairs, F., Elskens, M., Honda, M., Karl, D., Siegel, D., Silver, M., Steinberg, D., Valdes, J., Mooy, V., B, W., and S: Revisiting carbon flux through the ocean's twilight zone, Science, 316, 567-570, 2007.

Burd, A., Hansell, D., Steinberg, D., Anderson, T., Arístegui, J., Baltar, F., Beaupré, S., Buesseler, K., Dehairs, F., Jackson, G., Kadko, D., Koppelmann, R., Lampitt, R., Nagata, T., Reinthaler, T., Robinson, C., Robison, B., Tamburini, C., and Tanaka, T.: Assessing the apparent imbalance between geochemical and biochemical indicators of meso- and bathypelagic biological activity What the @ \$?! is wrong with present calculations of carbon budgets?, Deep-Sea Res. II, 57, 1557-1571, 2010.

Carlson, C., Hansell, D., Nelson, N., Siegel, D., Smethie, W., Katiwala, S., Meyers, M., Halewood, E.: Dissolved organic carbon export and subsequent remineralization in the mesopelagic and bathypelagic realms of the North Atlantic basin, Deep-Sea Res. II, 57, 1433-1445, 2010. 
Charland, M.: SigmaPlot 2000/2001 for Scientists, Riparian House Merrickville (Ontario), 2002.

Chen, J. and Strous, M.: Denitrification and aerobic respiration, hybrid electron transport chains and co-evolution, Biochim. Biophys. Acta, 1827, 136-144, 2013.

Christensen, J., Packard, T., Dortch, Q., Minas, H., Garfield, P., and Richez, C.: Carbon oxidation in the deep Mediterranean Sea: evidence for dissolved organic carbon source, Global Biogeochem. Cy., 3, 315-335, 1989.

Christensen, J. P. and Packard, T.: Respiratory electron transport activities in plankton: comparison of methods, Limnol. Oceanogr., 24, 576-583, 1979.

Cleland, W.: Enzyme kinetics, Ann. Rev. Biochem., 36.1, 77-112, 1967.

Codispoti, L. and Packard, T.: Denitrification rates in the Eastern tropical South Pacific, J. Mar. Res., 38, 453-477, 1980.

Codispoti, L., Dugdale, R., and Minas, A.: A comparison of the nutrient regimes off northwest Africa, Peru and Baja California, Rapp. P. Reun. Cons. Int. Explor. Mer., 180, 184-201, 1982.

Codispoti, L., Brandes, J., Christensen, J., Devol, A., Naqvi, S., Paerl, H., and Yoshinari, T.: The oceanic fixed nitrogen and nitrous oxide budhets: Moving targets as we enter the anthropocene?, Sci Mar, (Suppl. 2), 85-105, 2001.

Craig, H.: The deep metabolism: oxygen consumption in abyssal ocean water, J Geophys Res, 76, 5078-5086, 1971.

Dale, A. W., Sommer, S., Lomnitz, U., Montes, I., Treude, T., Liebetrau, V., Gier, J., Hensen, C., Dengler, M., Stolpovsky, K., Bryant, L. D., and Wallmann, K.: Organic carbon production, mineralisation and preservation on the Peruvian margin, Biogeosciences, 12, 1537-1559, doi:10.5194/bg-12-1537-2015, 2015.

Dalsgaard, T., Thamdrup, B., Farías, L., and Revsbech, N.: Anammox and denitrification in the oxygen minimum zone of the eastern South Pacific, Limnol. Oceanogr., 57, 1331-1346, 2012.

Ducklow, H. and Doney, S.: What is the metabolic state of the oligotrophic ocean? A debate, Annu. Rev. Mar. Sci., 5, 525-533, 2013.

Eppley, R. and Peterson, B.: Particulate organic matter flux and planktonic new production in the deep ocean, Nature, 282, 677680, 1979.

Ferguson, S.: ATP synthase: From sequence to ring size to the P/O ratio, Proc. Nati. Acad. Sci. USA, 107, 16755-16756, 2010.

Fernández, C., Faría, L., and Alcaman, M.: Primary production and nitrogen regeneration processes in surface waters of the Peruvian upwelling system, Prog. Oceanogr., 83, 159-168, 2009.

Fernández-Urruzola, I., Osma, N., Packard, T., Gómez, M., and Postel, L.: Distribution of zooplankton biomass and potential metabolic activities across the northern Benguela upwelling system, J. Mar. Syst., 140, 138-149, 2014.

Garfield, P., Packard, T., and Codispoti, L.: Particulate protein in the Peru upwelling system, Deep-Sea Res., 26, 623-639, 1979.

Giering, S., Sanders, R., Lampitt, R., Anderson, T., Tamburini, C., Boutrif, M., Zubkov, M., Marsay, C., Henson, S., Saw, K., Cook, K., and Mayor, D.: Reconciliation of the carbon budget in the ocean's twilight zone, Nature, 507, 480-483, 2014.

Gruber, N. and Sarmiento, J.: Global patterns of marine nitrogen fixation and denitrification, Global Biogeochem. Cy., 11, 235266, 1997.
Hafferty, A., Codispoti, L., and Huyer, A.: JOINT-I1 R/V Melville Legs I, I1 and IV WV Iselin Leg I1 bottle data March 1977-May 1977, 45, CUEA Data Rep., 1978.

Hansell, D., Carlson, C., and Schlitzer, R.: Net removal of major marine dissolved organic carbon fractions in the subsurface ocean, Global Biogeochem. Cy., 26, GB1016, doi:10.1029/2011GB004069, 2012.

Harvey, H.: The Chemistry and Fertility of Sea Waters. (Cambridge University Press, Cambridge), p. 224, 1955.

Jenkins, W. J.: Oxygen utilization rates in North Atlantic subtropical gyre and primary production in oligotrophic systems, Nature, 300, 246-248, 1982.

Jenkins, W. J.: The use of tracers and water masses to estimate rates of respiration, Heterotrophic Activity in the Sea, edited by: Hobbie, J. M., Williams, P. J., and Le, B., Plenum Press, New York, 391-403, 1984.

Jiao, N., Zhang, Y., Zhou, K., Li, Q., Dai, M., Liu, J., Guo, J., and Huang, B.: Revisiting the $\mathrm{CO}_{2}$ "source" problem in upwelling areas - a comparative study on eddy upwellings in the South China Sea, Biogeosciences, 11, 2465-2475, doi:10.5194/bg-11-24652014, 2014.

Karl, D.M.: Solar energy capture and transformation in the sea, Elem. Sci. Anthro., 2, 1-6, 2014.

Kenner, R. and Ahmed, S.: Measurements of electron transport activity in marine phytoplankton, Mar. Biol., 33, 119-127, 1975.

King, F., Devol, A., and Packard, T.: On plankton biomass and metabolic activity from the eastern tropical North Pacific, DeepSea Res., 25, 689-704, 1978.

King, L.: The coastal upwelling ecosystems analysis program as an experience in international cooperation, Ocean Dev. Int., 9, 269288, 1981.

Kogelshatz, J., Shepherd, R., Whitledge, T., Codispoti, L., and Huyer, A.: JOINT-II JASON 76 hydro data, R/V EASTWARD Cruises E-5F-76 through E-5L-76, International Decade of Ocean Exploration, CUEA Data Rep., vol. 38, 1978.

LaFerla, R., Azzaro, M., Civitarese, G., and Ribera d'Alcala, M.: Distribution patterns of carbon oxidation in the eastern Mediterranean Sea: evidence of changes in the remineralization processes, J. Geophys. Res., 108, 8111, doi:10.1029/2002JC001602, 2003.

Lane, N.: The Molecule that made the World, OUP (Oxford), p. 384, 2002.

Lane, N.: Power, Sex, Suicide: Mitochondria and the Meaning of Life, OUP (Oxford), p. 368, 2005.

Lane, N.: Life Ascending: The Ten Great Inventions of Evolution, OUP (Oxford), p. 352, 2009.

Lane, N., Allen, J., and Martin, W.: How did LUCA make a living? Chemiosmosis in the origin of life, BioEssays, 32, 271-280, 2010.

Laufkötter, C., Vogt, M., and Gruber, N.: Long-term trends in ocean plankton production and particle export between 1960-2006, Biogeosciences, 10, 7373-7393, doi:10.5194/bg-10-7373-2013, 2013.

Lotka, A.: Elements of physical biology, Williams and Wilkins Company, Baltimore, 495 pp., 1925.

MacIsaac, J., Dugdale, R., Barber, R., Blasco, D, and Packard, T.: Primary production cycle in an upwelling center, Deep-Sea Res., 32, 503-529, 1985. 
Madigan, M., Martinko, J., and Parker, J.: Brock Biology of Microorganisms, Prentice Hall, Upper Saddle River, New Jersey, p. 991, 2000

Martin, J. H., Knauer, G. A., Karl, D. M., and Broenkow, W. W.: VERTEX: carbon cycling in the northeast Pacific, Deep-Sea Res., 34, 267-285,1987.

Minas, J., Codispoti, L., and Dugdale, R.: Nutrients and primary production in the upwelling region off northwest Africa, Rapp. P. Reun. Cons. Int. Explor. Mer, 180, 148-183,1982.

Moran, L., Horton, R., Scrimgeour, K., and Perry, M.: Principles of Biochemistry, Prentice Hall, Saddle River, New Jersey, USA, 832 pp., 2012.

Munk, W.: Abyssal Recipes, Deep-Sea Res., 13, 707-730, 1966.

Nelson, D. and Cox, M.: Lehninger Principles of Biochemistry, Worth Publishers, New York, p. 1152, 2000.

Ochoa, S.: Efficiency of aerobic phosphorylation in cell-free heart extracts, J. Biol. Chem., 151, 493-505, 1943.

Odum, H. T.: Primary production in flowing waters, Limnol. Oceanogr., 1, 102-117, 1956.

Osma, N., Fernández-Urruzola, I., Packard, T., Postel, L., Gómez, M., and Pollehne, F.: Short-term patterns of vertical particle flux in northern Benguela: a comparison between sinking POC and respiratory carbon consumption, J. Mar. Syst., 140, 150-162, 2014.

Packard, T.: The estimation of the oxygen utilization rate in seawater from the activity of the respiration electron transport system in plankton, Ph.D. Thesis, University of Washington, Seattle, 1$115,1969$.

Packard, T.: Organizers remarks: Coastal Upwelling, in: Coastal and estuarine sciences 1, vol. xi-xiii, edited by: Richards, F. A., American Geophysiscal Union, Wasington DC, 1981.

Packard, T.: Oxygen consumption in the ocean: Measuring and mapping with enzyme analysis, in: Mapping strategies in chemical oceanography, edidet by: Zirino, A., Advances in Chemistry, American Chemical Society, Washington DC, 178-209, 1985a.

Packard, T.: Measurement of electron transport activity of marine microplankton, in: Advances in Aquatic Microbiology, edited by: Williams, L. and Jannasch, H., Academic, New York, 207-261, 1985b.

Packard, T. and Christensen, J.: Respiration and vertical carbon flux in the Gulf of Maine water column, J. Mar. Res., 62, 93-115, 2004.

Packard, T. and Codispoti, L.: Respiration, mineralization, and biochemical properties of the particulate matter in the southern Nansen Basin water column in April 1981, Deep-Sea Res. I, 54, 403-414, 2007.

Packard, T. and Gómez, M.: Modeling vertical carbon flux from zooplankton respiration, Prog. Oceanogr., 110, 59-68, 2013.

Packard, T. and Jones, V.: Biochemistry and ecology of the Peru Current: The JASON expedition to the Peru upwelling system, September 1976, CUEA Tech. Rep., 46, 129, 1976.

Packard, T. and Williams, P. LeB.: Rates of respiratory oxygen consumption and electron transport in surface seawater from the Northwest Atlantic Ocean, Oceanol. Ac., 4, 351-358, 1981.

Packard, T., Healy, M., and Richards, F.: Vertical distribution of the activity of the respiratory electron transport system in marine plankton, Limnol. Oceanogr., 16, 60-70, 1971.

Packard, T., Garfield, P., and Codispoti, L.: Oxygen consumption and denitrification below the peruvian upwelling, Coastal up- welling: Its sediment record, vol. 147-173, edited by: Suess, E. and Thiede, J., Plenum Press, New York, 147-173, 1983.

Packard, T., Blasco, D., and Dugdale, R.: Coastal Upwelling: A short summary of its physical, chemical and biological characteristics, in: Marine Geology and Oceanography of Arabian Sea and Coastal Pakistan, edited by: Haq, B. and Milliman, J., Van Nostrand Reinhold, New York, 339-350, 1984.

Packard, T., Denis, M., Rodier, M., and Garfield, P.: Deep-ocean metabolic $\mathrm{CO}_{2}$ production: calculations from ETS activity, Deep-Sea Res. I, 35, 371-382, 1988.

Pamatmat, M., Graf, G., Bengtsson, W., and Novak, C.: Heat production, ATP concentration and electron transport activity of marine sediments, Mar. Ecol. Prog. Ser., 4, 135-143, 1981.

Redfield, A., Ketchum, B., and Richards, F.: The influence of organisms on the composition of seawater, edited by: Hill, N. M., The Seas vol. II, Interscience New York, 26-77, 1963.

Richards, F.: Oxygen in the Ocean, in: Treatise on Marine Ecology and Paleoecology, edited by: Hedgepeth, J. W., Geol. Soc. America., 67, 185-238, 1957.

Richards, F.: Anoxic Basins and Fjords, in: Chemical Oceanography, edited by: Riley, J. P. and Skirrow, G., Academic Press, New York, 611-645, 1965.

Richards, F.: Coastal upwelling, American Geophysical Union, Washington, DC, p. 529, 1981.

Riley, G.: Oxygen, phosphate, and nitrate in the Atlantic Ocean, Bingham Oceanogr. Collection Bull., 13, 1-169, 1951.

Rykaczewski, R. and Checkley, D.: Influence of ocean winds on the pelagic ecosystem in upwelling regions, PNAS, 105, 1065-1970, 2008.

Ryther, J., Menzel, D., Hulburt, E., Lorenzen, C., and Corwin, N.: Production and utilization of organic matter in the Peru Coastal Current, Anton. Brun. Rep., 4, 4.3-4.12, 1970.

Santoso, A., McGregor, S., Jin, F., Cai, W., England, M., An, S., McPhaden, M., and Guilyardi, E.: Late-twentieth-century emergence of the El Niño propagation asymmetry and future projections, Nature, 504, 126-130, 2013.

Seiwell, H.: The distribution of oxygen in the western basin of the North Atlantic, Papers in Phys. Ocean. and Meteorol, VoL. IIi, p. 1-86, 1934.

Seiwell, H.: Consumption of oxygen in seawater under controlled laboratory conditions, Nature, 140, 506-507, 1937.

Smith, R.: Upwelling, Oceanogr. Mar. Biol. Annu. Rev., 6, 11-46, 1968.

Smolders, G. J. F., van der Meij, J. , van Loosdrecht, M. C. M., and Heijnen, J. J.: Stoichiometric model of the aerobic metabolism of the biological phosphorus removal process, Biotech. Bioeng., 44, 837-848, 1994.

Suess, E.: Particulate organic carbon flux in the oceans-surface productivity and oxygen utilization, Nature, 288, 260-263, 1980.

Takahashi, T., Broecker, W., and Langer, S.: Redfield ratio based on chemical data from isopycnal surfaces, J. Geophys. Res., 90, 6907-6924, 1985.

van Loosdrecht, MCM, S., GJ, K., T, H., and JJ: Metabolism of micro-organisms responsible for enhanced biological phosphorus removal from wastewater, Antonie Leeuwenhoek, 71, 109116, 1997.

Walker, J.: ATP synthesis by rotary catalysis, Angew. Chem. Int. Ed., 37, 2308-2319, 1998. 
Walsh, J.: Implications of a systems approach to oceanography, Science, 176, 969-975, 1972.

Walsh, J., Kelly, J., Dugdale, R., and Frost, B.: Implications of a systems approach to oceanography, Gross features of the Peruvian upwelling system with special reference to possible diel variation, Invest. Pesq., 35, 25-42, 1971.

Watt, N., Montgomery, M., Runswick, M., Leslie, A., and Walker, J.: Bioenergetic cost of making an adenosine triphosphate molecule in animal mitochondria, PNAS, 107, 16823-16827, 2010 .
Williams, P., Quay, P., Westberry, T., and Behrenfeld, M.: The oligotrophic ocean is autotrophic, Annu. Rev. Mar. Sci., 16.1-16.15, 2012.

Wilson, S., Kolber, Z., Tozzi, S., Zehr, J., and Karl, D.: Nitrogen fixation, hydrogen cycling, and electron transport kinetics in trichodesmium erythraeum (cyanobacteria) strain ims1011, J. Phycol. 48, 595-606, 2012.

Wooster, W.: Yearly changes in the Peru Current, Limnol. Oceanogr., 6, 222-226, 1961.

Wyrtki, K.: Circulation and water masses in the Eastern equatorial Pacific Ocean, Int. J. Oceanol. Limnol., 1, 117-147, 1967.

Zheng, Y, Schlosser, P., Swift, J., and Jones, E.: Oxygen utilization rates in the Nansen Basin, Arctic Ocean: implications for new production, Deep-Sea Res., 144, 1923-1943, 1997. 